\title{
Low-temperature binding of NO adsorbed on MIL-100(AI)-A case study for the application of high resolution pulsed EPR methods and DFT calculations
}

Matthias Mendt, Benjamin Barth, Martin Hartmann, and Andreas Pöppl

Citation: The Journal of Chemical Physics 147, 224701 (2017); doi: 10.1063/1.4995551

View online: https://doi.org/10.1063/1.4995551

View Table of Contents: http://aip.scitation.org/toc/jcp/147/22

Published by the American Institute of Physics

\section{Articles you may be interested in}

A climbing image nudged elastic band method for finding saddle points and minimum energy paths

The Journal of Chemical Physics 113, 9901 (2000); 10.1063/1.1329672

Quantum confinement effects on electronic photomobilities at nanostructured semiconductor surfaces:

$\mathrm{Si}(111)$ without and with adsorbed Ag clusters

The Journal of Chemical Physics 147, 224703 (2017); 10.1063/1.4999943

Characterizing the geometric and electronic structure of defects in the "29" copper surface oxide

The Journal of Chemical Physics 147, 224706 (2017); 10.1063/1.4996729

Resonance Raman excitation profiles of CdS in pure CdS and CdSe/CdS core/shell quantum dots: CdSlocalized excitons

The Journal of Chemical Physics 147, 224702 (2017); 10.1063/1.5003099

Cholesteric and screw-like nematic phases in systems of helical particles

The Journal of Chemical Physics 147, 224903 (2017); 10.1063/1.4996610

Perspective: Aerosol microphysics: From molecules to the chemical physics of aerosols

The Journal of Chemical Physics 147, 220901 (2017); 10.1063/1.5002641

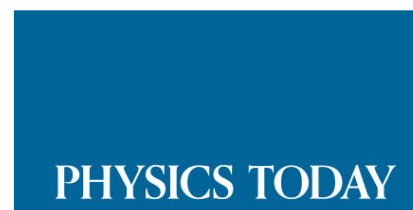

WHITEPAPERS
ADVANCED LIGHT CURE ADHESIVES

Take a closer look at what these environmentally friendly adhesive systems can do

\section{READ NOW}

PRESENTED BY

Q MASTERBOND: 


\title{
Low-temperature binding of NO adsorbed on MIL-100(Al)_A case study for the application of high resolution pulsed EPR methods and DFT calculations
}

\author{
Matthias Mendt, ${ }^{1}$ Benjamin Barth, ${ }^{2}$ Martin Hartmann, ${ }^{2}$ and Andreas Pöppl ${ }^{1}$ \\ ${ }^{1}$ Felix-Bloch-Institut für Festkörperphysik, Universität Leipzig, Linnéstr. 5, 04103 Leipzig, Germany \\ ${ }^{2}$ Erlangen Catalysis Resource Center, Friedrich-Alexander-Universität Erlangen-Nürnberg, Egerlandstr. 3, \\ 91058 Erlangen, Germany
}

(Received 12 July 2017; accepted 20 November 2017; published online 8 December 2017)

\begin{abstract}
The low-temperature binding of nitric oxide (NO) in the metal-organic framework MIL-100(Al) has been investigated by pulsed electron nuclear double resonance and hyperfine sublevel correlation spectroscopy. Three NO adsorption species have been identified. Among them, one species has been verified experimentally to bind directly to an ${ }^{27} \mathrm{Al}$ atom and all its relevant ${ }^{14} \mathrm{~N}$ and ${ }^{27} \mathrm{Al}$ hyperfine interaction parameters have been determined spectroscopically. Those parameters fit well to the calculated ones of a theoretical cluster model, which was derived by density functional theory (DFT) in the present work and describes the low temperature binding of NO to the regular coordinatively unsaturated $\mathrm{Al}^{3+}$ site of the MIL-100(Al) structure. As a result, the Lewis acidity of that site has been characterized using the NO molecule as an electron paramagnetic resonance active probe. The DFT derived wave function analysis revealed a bent end-on coordination of the NO molecule adsorbed at that site which is almost purely ionic and has a weak binding energy. The calculated flat potential energy surface of this species indicates the ability of the NO molecule to freely rotate at intermediate temperatures while it is still binding to the $\mathrm{Al}^{3+}$ site. For the other two NO adsorption species, no structural models could be derived, but one of them is indicated to be adsorbed at the organic part of the metal-organic framework. Hyperfine interactions with protons, weakly coupled to the observed NO adsorption species, have also been measured by pulsed electron paramagnetic resonance and found to be consistent with their attribution to protons of the MIL-100(Al) benzenetricarboxylate ligand molecules. Published by AIP Publishing. https://doi.org/10.1063/1.4995551
\end{abstract}

\section{INTRODUCTION}

Metal-organic frameworks (MOFs) are crystalline porous compounds built up from inorganic metal-oxygen clusters that are connected by organic ligands. ${ }^{1,2}$ Due to their unique and varied structural and physiochemical properties, they promise applications in different areas including adsorption and catalysis. $^{3-7}$ Trivalent aluminum based MOFs with coordinatively unsaturated metal sites (CUSs) are particularly interesting for heterogeneous catalysis since $\mathrm{Al}^{3+}$ sites are known to be strong Lewis acids. ${ }^{8-12}$ In addition, Al-based MOFs show extraordinary thermal and chemical stability due to their strong metaloxygen bonds ${ }^{8,13}$ and are low in price, ${ }^{8}$ making them favorable for industrial applications. Consequently, the spectroscopic characterization of Lewis acid sites in Al-based MOFs is of high relevance.

The Lewis acidity of cationic sites can be characterized by electron paramagnetic resonance (EPR) spectroscopy, using nitric oxide (NO) after its adsorption at such sites as an EPR active probe. This approach has been successfully applied for the characterization of Lewis acid sites in various zeolites. ${ }^{11,14,15}$ The small molecule NO has one unpaired electron in its anti-bonding $\pi^{*}$ molecular orbital (MO) ${ }^{16-19}$ which is responsible for a characteristic EPR signal of NO adsorbed at a Lewis acid site. ${ }^{11,14,15,20}$ The EPR derived ${ }^{14} \mathrm{~N}$ hyperfine interaction (hfi) of such a species allows a quantitative characterization of the corresponding Lewis acidity. ${ }^{11,14,15}$

Lewis acid sites in MOFs are often characterized experimentally by infrared (IR) spectroscopy, temperatureprogrammed desorption, or the determination of the isosteric heats of adsorption of certain probe molecules. ${ }^{21}$ For example, some authors investigated the strength of the Lewis acid sites in some MIL-100(M) $(\mathrm{M}=$ trivalent metal, $\mathrm{MIL}=$ Materials of Institute Lavoisier) MOFs by IR spectroscopy using CO, pyridine, and acetonitrile as probe molecules. ${ }^{9,22-24}$ Nevertheless, probing Lewis acidity by the afore-mentioned techniques has the disadvantage in that the nature of the Lewis acid sites might not be derived unambiguously from those methods alone. Their attribution to other adsorption sites rather than the regular CUS cannot be fully excluded in some cases. Characterizing Lewis acidity by EPR, using NO as a probe, will overcome this disadvantage for Al-based MOFs since the EPR signal of the NO adsorption species reveals unambiguously the interaction with the corresponding Al site via the ${ }^{27} \mathrm{Al}$ hfi. ${ }^{25}$ The analysis of the EPR signature of such an NO adsorption species, aided by first principle calculations like density functional theory (DFT), will allow the determination of the nature of the Lewis acid site, characterizing its electron acceptor strength at the same time by the $\mathrm{NO}{ }^{14} \mathrm{~N}$ hfi. The present 
a)

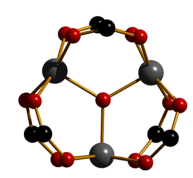

b)

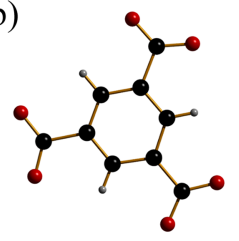

c)

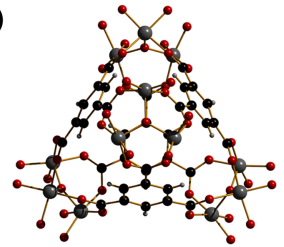

d)

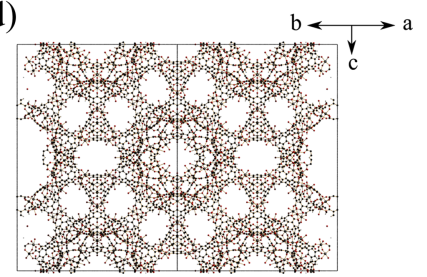

FIG. 1. Structural components of the MIL-100(Al) material as taken from the work of Volkringer et al.: ${ }^{26}$ The $\mu_{3}$-oxo-bridged $\mathrm{Al}_{3} \mathrm{O}$ building unit (a) without showing the water molecules and the anion coordinating to the CUS, and the btc ligand molecule (b). Both form a supertetrahedral block (c) in which the corners are occupied by the trimeric metal unit. This supertetrahedral building block is further connected to the MTN topology (d) as it has the zeolite ZSM-39 (Zeolite Socony Mobil - thirty nine). ${ }^{26}$ The lines in (d) border the unit cell. The atoms are colored in light gray $(\mathrm{H})$, black $(\mathrm{C})$, red $(\mathrm{O})$, and dark gray (Al)

work is a case study for this approach, demonstrating that a combination of high-resolution pulsed EPR methods applied to NO adsorbed on an Al-based MOF and DFT calculations on appropriate cluster models can indeed reach the desired goal.

We have chosen the MOF MIL-100( $\mathrm{Al})^{26}$ as the model system for the present study. The inorganic connectors of this MOF consist of $\mu_{3}$-oxo-bridged $\mathrm{Al}^{3+}$ octahedra, which are linked by organic benzenetricarboxylate (btc) ligands leading to a mesoporous cage-type pore structure with the sum formula $\mathrm{Al}_{3} \mathrm{O}(\mathrm{btc})_{2}(\mathrm{X})\left(\mathrm{H}_{2} \mathrm{O}\right)_{2}(\mathrm{X}=\mathrm{F}, \mathrm{OH})$ (see Fig. 1). ${ }^{26}$ After a thermal treatment, water molecules coupled to the metal ions are removed, producing the CUS. ${ }^{8}$ This material has two giant cavities having free diameters of about 2.5 $\mathrm{nm}$ and $2.9 \mathrm{~nm},{ }^{27}$ which might also allow its use as a host for encapsulated metal complexes, e.g., in catalysis. ${ }^{28}$ The MIL-100(Al) MOF also induces no significant in vitro cell toxicity, ${ }^{29}$ which is an interesting property with respect to potential medical applications eventually using this material as a drug carrier. ${ }^{30}$ It is worth mentioning, in this context, that the small molecule NO plays unique physiological roles in various biological systems of human, mammal, and even plants. ${ }^{31-40}$ Thus the development of suitable NO drug-delivery systems using porous materials as NO storage devices is a research field of high medical interest ${ }^{41-49}$ including the consideration of MOFs as possible NO carriers. ${ }^{50-58}$ Several MOFs with CUS have been shown to adsorb large amounts of NO at room temperature and to release it under humid conditions. ${ }^{50,53,54,56}$ In particular, this has been demonstrated for the MOF MIL-100(Fe), which promotes unsaturated $\mathrm{Fe}^{3+}$ sites for NO chemisorption. ${ }^{55}$ Recently we have shown that its $\mathrm{Al}^{3+}$ based version MIL-100(Al) ${ }^{26}$ binds NO much weaker than MIL-100(Fe), ${ }^{25}$ a difference which has not been investigated on an atomistic scale yet. One part of such a study, namely the determination of the microscopic geometric and electronic structure of the $\mathrm{NO}$ adsorption species at the regular $\mathrm{Al}^{3+}$ CUS of the MIL-100(Al) material, is also addressed

by the present work by results obtained from DFT calculations, which are experimentally verified by specific pulsed EPR experiments.

\section{EXPERIMENTAL METHODS}

\section{Sample preparation}

The MOF material MIL-100(Al) has been synthesized by standard procedures and characterized as described elsewhere. ${ }^{25}$ For the preparation of the EPR samples, $9.0 \mathrm{mg}$ of MIL-100(Al) was filled into a conventional X-band EPR quartz glass tube. The tubes were connected to a stainless steel valve that was fitted to a stainless steel vacuum line. The samples were activated at $T=443 \mathrm{~K}$ for about $18 \mathrm{~h}$ under dynamic vacuum at pressures $p<10^{-4}$ mbar. Then, $2.8 \pm 0.3 \mu \mathrm{mol}$ of NO ( $99.5 \%$ purity) was condensed into the EPR tubes applying a cold trap with liquid nitrogen. The EPR tubes were sealed while applying the cold trap, which ensured that the total amount of NO remained in the EPR tubes during sealing. The latter was proven by appropriate pressure measurements of the NO gas remaining in the stainless steel valve which ensured that more than $99 \%$ of the NO gas had been trapped within the EPR tubes.

\section{Spectroscopic methods}

Two pulse electron spin echo (ESE) ${ }^{59}$ field sweep experiments, two-dimensional (2D) hyperfine sublevel correlation (HYSCORE) experiments, ${ }^{59,60}$ and Davies electron nuclear double resonance (ENDOR) ${ }^{59}$ experiments were performed on a Bruker ELEXYS E580 X-band $(9.75 \mathrm{GHz})$ spectrometer equipped with an ER411X-MD-5 dielectric pulse ENDOR resonator and an Oxford Instruments CF935 cryostat. All experiments were performed at temperatures $5 \mathrm{~K}<T<7 \mathrm{~K}$ with a short repetition time of $2 \mathrm{~ms}$ for the ENDOR experiment and of $1 \mathrm{~ms}$ for the ESE and HYSCORE experiments.

The ESE field sweep experiments were conducted with the pulse sequence $\pi / 2-\tau-\pi-\tau-$ echo where the time delay between both pulses was chosen to be $\tau=140 \mathrm{~ns}$ or $\tau=1200 \mathrm{~ns}$. In the first case, the $\pi / 2$ and $\pi$ pulses had lengths of $16 \mathrm{~ns}$ and $32 \mathrm{~ns}$, whereas in the second case, selective pulses with lengths of $100 \mathrm{~ns}$ and $200 \mathrm{~ns}$ have been applied, reproducing the conditions of the HYSCORE and ENDOR experiments, respectively.

The HYSCORE experiment pulse sequence was $\pi / 2-\tau$ $-\pi / 2-t_{1}-\pi-t_{2}-\pi / 2-\tau-$ echo, where the time variables $t_{1}$ and $t_{2}$ were varied independently keeping the time delay $\tau$ constant. The latter was adjusted differently in different measurements to avoid distinct blind spots for the different nuclear ${ }^{27} \mathrm{Al}$ and ${ }^{1} \mathrm{H}$ HYSCORE signals. ${ }^{61}$ Appropriate values of $\tau$ for the detection of a strongly coupled ${ }^{27} \mathrm{Al}$ species were determined from the blind spot behavior of the HYSCORE sequence as shown in Fig. S1 of the supplementary material. The time delays $t_{1}$ and $t_{2}$ were increased in time steps $\Delta t=24 \mathrm{~ns}$ starting at $t_{1,0}=t_{2,0}=80 \mathrm{~ns}$ if nothing else is stated. The $\pi / 2$ and $\pi$ pulses had lengths of $16 \mathrm{~ns}$ and $32 \mathrm{~ns}$, respectively. In that way, $170 \times 1702 \mathrm{D}$ time domain spectra of the HYSCORE echo intensity were recorded. After third-order polynomial base line corrections and zero-filling to 
$1024 \times 1024$ points, the frequency domain HYSCORE spectra with frequency coordinates $v_{1}$ and $v_{2}$ were obtained as the magnitudes of the corresponding 2D Fourier transforms.

For the ENDOR experiments, the Davies type micro wave (mw) pulse sequence $\pi-t-\pi / 2-\tau-\pi-\tau-$ echo was chosen, and during the $t=14 \mu \mathrm{s}$ mixing period, a radio frequency pulse with length $10 \mu$ s was applied. The selective $\pi / 2$ and $\pi$ pulses had lengths of $100 \mathrm{~ns}$ and $200 \mathrm{~ns}$, respectively, and a time delay $\tau=1200 \mathrm{~ns}$ was set for the primary echo sequence which reads out the polarization of the electron spin observer transition. ${ }^{59}$

\section{Spectral analysis}

The ${ }^{2} \Pi_{1 / 2}$ ground state of gaseous NO is diamagnetic due to the cancelation of spin and orbital momentum. ${ }^{17}$ But if $\mathrm{NO}$ is adsorbed at some surface site, the electric field gradient at this site can quench the orbital momentum and the ${ }^{2} \Pi_{1 / 2}$ ground state becomes paramagnetic having an electron spin $S=1 / 2$ and $g$-values near but distinctly smaller than the free electron g-value $g_{e}=2.0023 .^{20}$ The magnetic parameters of such an NO adsorption species can be described by the spin Hamiltonian $^{62}$

$$
\hat{H}=\mu_{\mathrm{B}} \boldsymbol{B}_{0}^{T} \boldsymbol{g} \hat{\boldsymbol{S}}+\sum_{i} \hat{\boldsymbol{S}}^{\mathrm{T}} \boldsymbol{A}_{\boldsymbol{i}} \hat{\boldsymbol{I}}_{\boldsymbol{i}}-\sum_{i} \mu_{\mathrm{n}} g_{\mathrm{n}, i} \boldsymbol{B}_{0}^{\mathrm{T}} \hat{\boldsymbol{I}}_{\boldsymbol{i}}+\sum_{j} \hat{\boldsymbol{I}}_{j}^{\mathrm{T}} \boldsymbol{Q}_{j} \hat{\boldsymbol{I}}_{\boldsymbol{j}}
$$

The electron Zeeman interaction term is expressed by the vector operator $\hat{\boldsymbol{S}}$ describing the electronic spin $S$ of the NO molecule, the external magnetic field vector $\boldsymbol{B}_{0}^{T}$, the g-tensor $\boldsymbol{g}$ with principle values $g_{x x}>g_{y y}>g_{z z}$, and the Bohr magneton $\mu_{\mathrm{B}}$.

The hyperfine interaction (hfi) between the electron spin and the i-th neighbored nuclear spin $I_{i}$, expressed by the vector operator $\hat{\boldsymbol{I}}_{i}$, is described by the tensors $\boldsymbol{A}_{i}$ with principle values $A_{x x}, A_{y y}$, and $A_{z z}$. In its principle axis system, such a tensor can be redefined according to the formula

$$
\boldsymbol{A}=a_{\mathrm{iso}} \mathbf{1}+T\left(\begin{array}{ccc}
-(1+\rho) & 0 & 0 \\
0 & -(1-\rho) & 0 \\
0 & 0 & 2
\end{array}\right)
$$

where $a_{\text {iso }}$ is the isotropic Fermi contact coupling constant, $T$ is the hfi dipolar coupling constant, $\rho$ is the orthorhombicity parameter, and $\mathbf{1}$ is the identity matrix.

The third term in Eq. (1) expresses the nuclear Zeeman interaction with the nuclear g-factors $g_{\mathrm{n}, i}$ and $\mu_{\mathrm{n}}$ being the nuclear magneton.

The ${ }^{27} \mathrm{Al}$ and ${ }^{14} \mathrm{~N}$ nuclei have nuclear spins ${ }^{27 \mathrm{Al}} I=5 / 2$ and ${ }^{14 \mathrm{~N}} I=1$. As a consequence, these nuclei interact with the electric field gradient at their positions which is expressed by the last term in Eq. (1), where $\boldsymbol{Q}_{j}$ are the nuclear quadrupole interaction (nqi) tensors. In its principle axis systems, such a tensor can be expressed as

$$
\boldsymbol{Q}=\frac{C_{Q}}{4 I(2 I-1)}\left(\begin{array}{ccc}
-(1+\eta) & 0 & 0 \\
0 & -(1-\eta) & 0 \\
0 & 0 & 2
\end{array}\right)
$$

The parameter $C_{Q}$ is the quadrupole coupling constant, $\eta$ is the biaxiality of the electric field gradient tensor, and $I$ is the spin quantum number of the corresponding nuclear spin.

Consider an electron spin $S=1 / 2$ coupled to a nuclear spin $I$. The Zeeman interaction splits all energy levels defined as eigenvalues of the Hamiltonian in Eq. (1) into $\alpha$ and $\beta$ submanifolds having electronic magnetic quantum numbers $m_{S}$ $= \pm 1 / 2$ in the high field limit, respectively. In the presence of the nuclear Zeeman interaction, each $\alpha$ and $\beta$ manifold splits further into $2 I+1$ equally distant levels with energies $\varepsilon\left(m_{S}, m_{I}\right)$ which are additionally characterized by the nuclear magnetic quantum numbers $m_{I}=-I,-I+1, \ldots, I-1, I$ in an approximate fashion and which are further altered by the nqi if $I \geq 1$. For those energies, one derives the first-order equations 59

$$
\begin{aligned}
\varepsilon^{1}\left(m_{S}, m_{I}\right)= & v_{S} m_{S}+c\left(m_{S}\right) m_{I} \\
& -\frac{1}{2}\left[I(I+1)-3 m_{I}^{2}\right] Q_{z z}^{\prime}\left(m_{S}\right),
\end{aligned}
$$

where $v_{S}$ is the electron's Lamor frequency. The parameter $c\left(m_{S}\right)$ depends on $m_{S}$, the full $\mathrm{g}$ - and hfi-tensors, the nuclear Lamor frequency $v_{I}$, and the direction of the external magnetic field. ${ }^{59}$ But it is independent of the magnetic quantum number $m_{I}$. The parameter $Q_{z z}^{\prime}\left(m_{S}\right)$ is the zz-matrix element of a suitably transformed nqi-tensor. ${ }^{59}$ It depends on the latter and further on the same parameters as $c\left(m_{S}\right)$. In particular, it is independent of $m_{I}$. The energy differences between two levels within the same $\alpha$ or $\beta$ manifold but different nuclear magnetic quantum numbers $m_{I}$ and $m_{I}^{\prime}$ translate into the nuclear frequencies $v_{m_{I}, m_{I}^{\prime}}^{\alpha, \beta}$. These frequencies are altered by the hfi and nqi and consequently contain the information about the respective tensors. ${ }^{59}$ It follows from Eq. (4) that all frequencies $v_{m_{I}, m_{I}^{\prime}}^{\alpha, \beta}$ depend on the nqi in first order as long as $\left|m_{I}\right| \neq\left|m_{I}^{\prime}\right|$. Otherwise they are independent on the nqi in first order. In particular, in the case of non-integer nuclear spin $I>1$, the

\begin{tabular}{|c|c|c|c|c|c|c|c|}
\hline $\begin{array}{l}\text { NO adsorption } \\
\text { species }\end{array}$ & $\begin{array}{l}\text { Relative } \\
\text { amount }\end{array}$ & $g_{x x}$ & $g_{y y}$ & $g_{z z}$ & $\begin{array}{l}\left|A_{x x}^{14 \mathrm{~N}}\right| \\
(\mathrm{MHz})\end{array}$ & $\begin{array}{l}\left|A_{y y}^{14 \mathrm{~N}}\right| \\
(\mathrm{MHz})\end{array}$ & $\begin{array}{l}\left|A_{z z}^{14 \mathrm{~N}}\right| \\
(\mathrm{MHz})\end{array}$ \\
\hline $\mathrm{NOa}$ & 1 & $1.997(3)$ & $1.997(3)$ & $1.909(3)$ & $14(8)$ & $86(3)^{\mathrm{a}}$ & $20(6)$ \\
\hline $\mathrm{NOb}$ & $0.8(6)$ & $1.992(11)$ & $1.992(11)$ & $1.88(1)$ & $\ldots{ }^{b}$ & $\ldots{ }^{b}$ & $\ldots{ }^{b}$ \\
\hline NOc & $<4.3$ & 1.97(3) & $1.97(3)$ & $1.4 \ldots 1.88^{c}$ & $\ldots{ }^{b}$ & $\ldots{ }^{b}$ & $\ldots{ }^{b}$ \\
\hline
\end{tabular}
central transition frequencies $v_{\alpha, \beta}:=v_{\frac{1}{2},-\frac{1}{2}}^{\alpha, \beta}$ depend on the nqi only in second order. ${ }^{59}$

TABLE I. Experimentally derived relative amounts, g-tensor principle values, and the ${ }^{14} \mathrm{~N}$ hfi principle values of the different NO adsorption species in MIL-100(Al).

${ }^{\mathrm{a}}$ Data from the work of Barth et al. ${ }^{25}$

${ }^{\mathrm{b}}$ Not resolved.

${ }^{\mathrm{c}} \mathrm{NOc}$ might reflect a variety of weakly physisorbed NO species with $g_{z z}$-values distributed within the given range. 
TABLE II. ${ }^{27} \mathrm{Al}$ hfi and nqi parameters as determined experimentally by Davies ENDOR and HYSCORE spectroscopy for the ${ }^{27} \mathrm{Al}$ species Ala and by HYSCORE spectroscopy for the ${ }^{27} \mathrm{Al}$ species Alb and Alc, where $\alpha$ and $\beta$ are the Euler angles of the hfi tensors. Since all nuclei show an almost axially symmetric hfi tensor within the experimental resolution, the determination of the third Euler angle $\gamma$ was not possible in all cases.

\begin{tabular}{cccccccc}
\hline \hline & $\begin{array}{c}a_{\text {iso }} \\
(\mathrm{MHz})\end{array}$ & $\begin{array}{c}T \\
(\mathrm{MHz})\end{array}$ & $|\rho|$ & $\alpha(\mathrm{deg})$ & $\beta(\mathrm{deg})$ & $\begin{array}{c}C_{Q} \\
(\mathrm{MHz})\end{array}$ & $\eta$ \\
\hline Ala & $14.7(17)$ & $3.4(5)$ & $<0.5^{\mathrm{a}}$ & $90(30)$ & $50(10)$ & $\ldots{ }^{\mathrm{b}}$ & $\ldots{ }^{\mathrm{b}}$ \\
Alb & $-4.2(6)$ & $1.4(6)$ & $\ldots{ }^{\mathrm{b}}$ & $\ldots{ }^{\mathrm{b}}$ & $55(35)$ & $2.8(20)$ & $\ldots{ }^{\mathrm{b}}$ \\
Alc & $|\cdot|<0.3^{\mathrm{c}}$ & $<0.3^{\mathrm{c}}$ & $\ldots{ }^{\mathrm{b}}$ & $\ldots{ }^{\mathrm{b}}$ & $\ldots{ }^{\mathrm{b}}$ & $5.2(8)^{\mathrm{c}}$ & $0.26(26)^{\mathrm{c}}$ \\
\hline \hline
\end{tabular}

${ }^{\mathrm{a}}$ Within the resolution of Davies ENDOR spectroscopy.

${ }^{\mathrm{b}}$ Not resolved.

${ }^{\mathrm{c}}$ This is a mean value of a large number of different distant nuclei.

Spectral simulations were realized in the present work with the MatLab toolbox EasySpin ${ }^{62}$ carrying out the exact diagonalization of the spin Hamiltonian in Eq. (1). The proposed inhomogeneous linewidth model for the simulated ENDOR and HYSCORE signals of strongly coupled ${ }^{27} \mathrm{Al}$ was implemented by the summation of 1040 and 9312 simulated time domain signals, respectively, where distinct parameters were assumed to be independently Gaussian distributed random numbers. Resulting deviations of the simulated from the experimental spectra reflect the restricted distribution model assumed for the simulation parameters and are considered by the experimental errors of the determined spin-Hamiltonian parameters summarized in Tables I and II. The HYSCORE spectra of distant ${ }^{27} \mathrm{Al}$ nuclei were calculated as a sum of 256 powder spectra according to the spherical-averaging approximation. ${ }^{63}$ Here the Euler angles between the hfi and nqi tensors were treated as uniformly distributed random numbers within their non-redundant full ranges. In general, Euler angles of the various tensors in this paper are given relatively to the g-tensor frame as used by EasySpin. ${ }^{62}$ For all experimental and simulated HYSCORE spectra, the sum projections are given on the top and at the right. All HYSCORE simulations have been normalized to the maximum intensity of the corresponding experimental signal and have the same contour number and range. All contour plots are scaled logarithmically.

\section{COMPUTATIONAL DETAILS}

\section{Model systems}

DFT calculations were performed for certain cluster models. All clusters include the $\left[\mathrm{Al}_{3}\left(\mu_{3}-\mathrm{O}\right)\right]^{7+}$ core of the MIL-100(Al) [Fig. 1(a)]. Some clusters were saturated with six 1,3,5-btc ligand ions with the sum formula $\left[\mathrm{C}_{9} \mathrm{O}_{6} \mathrm{H}_{3}\right]^{3-}$ [Fig. 1(b)]. Such a starting cluster was cut out from the MIL$100(\mathrm{Al})$ framework structure as it was determined by singlecrystal X-ray microdiffraction. ${ }^{26}$ Here we have to note that the MIL-100(Al) crystal structure has seven non-equivalent aluminum sites. ${ }^{26}$ But the differences between their local geometric structures are only small, ${ }^{26}$ and thus, we claim that the cluster we have cut out is representative. The outermost twelve oxygen atoms of the two non-coordinated carboxylate groups of each btc ligand were saturated with hydrogen atoms [as shown in Figs. 2(a)-2(c)]. We call this structural model ML0. During all geometry optimizations, the 24 oxygen atoms of the non-coordinated carboxylate groups of the btc ligands were constrained to the coordinates of the starting crystal structure. ${ }^{26}$ All other atoms including the twelve added hydrogen atoms and additional ligand molecules at the $\mathrm{Al}^{3+}$ CUS of other structural models were allowed to fully relax if nothing else is stated. a)

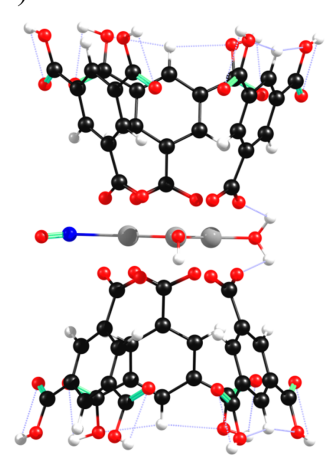

d)

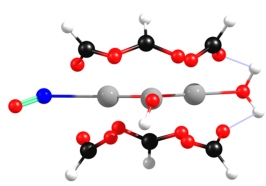

b)

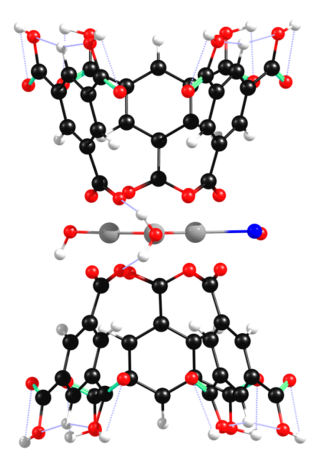

e)

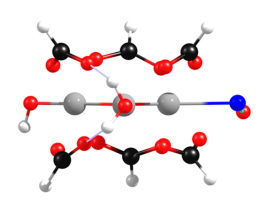

g)

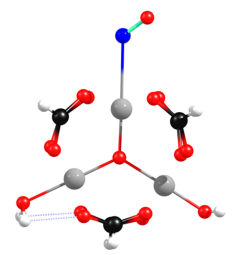

c)

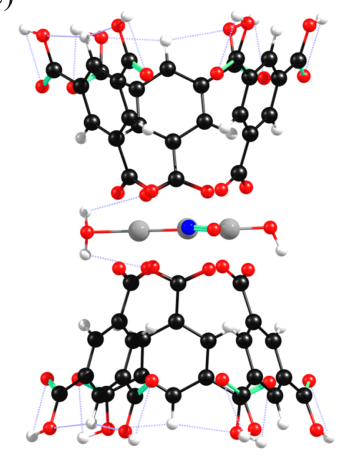

f)

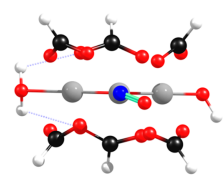

FIG. 2. Different views of the final cluster models ML3 [(a)-(c)] and M3 [(d)-(g)] optimized at the B3LYP/def2TZVP level. The atoms are colored in white $(\mathrm{H})$, black $(\mathrm{C})$, blue $(\mathrm{N})$, red $(\mathrm{O})$, and gray $(\mathrm{Al})$. 
The cluster MLO has a net charge of +1 . For charge compensation, we add an $\mathrm{OH}^{-}$ion ${ }^{8,26}$ at one CUS of the MLO cluster obtaining the electrical neutral structural model ML1. Since it was indicated by solid state nuclear magnetic resonance (NMR) spectroscopy that one water molecule remains adsorbed at one $\mathrm{Al}^{3+}$ of each $\mathrm{Al}_{3}\left(\mu_{3}-\mathrm{O}\right)$ cluster during activation, ${ }^{8}$ we construct the model ML2 by adding a water molecule to one CUS of the geometrical optimized model ML1. The structural model ML3 [Figs. 2(a)-2(c)] was obtained by adding an NO molecule at the remaining CUS of the optimized model ML2.

Calculations were also performed for smaller clusters without the btc ligands including again the $\left[\mathrm{Al}_{3}\left(\mu_{3}-\mathrm{O}\right)(\mathrm{OOC})_{6}\right]^{+}$unit that was cut from the MLO structure. The open bonds at the carbon atoms were saturated by hydrogen atoms [as shown in Figs. 2(d)-2(g)] leading to the structural model M0. Adding additional ligand atoms at the CUS were obtained analogously to the MLX model series [as shown in Figs. 2(d)-2(g)]. The corresponding models without btc ligands are called $\mathbf{M X}$, where $\mathbf{X}$ goes from 1 to 3 . The geometry optimizations of the models of the $\mathbf{M X}$ series were performed without any geometrical constraints if nothing else is stated.

For all model clusters, we label the $\mathrm{Al}^{3+}$ ion, where NO binds by Al1, where water binds by $\mathrm{Al} 2$, and where the $\mathrm{OH}^{-}$ binds by $\mathrm{Al} 3$.

\section{Computational methods}

All calculations were performed with the ORCA 3.0.0 program package. ${ }^{64}$ For all models with multiplicity 1 , the restricted Kohn-Sham formalism was used, whereas models with multiplicity 2 were treated with the unrestricted KohnSham formalism. ${ }^{64}$ The models ML1, M1, ML2, and M2 were geometry optimized using the B3LYP ${ }^{65,66}$ functional together with the def2-TZVP ${ }^{67,68}$ basis set. For that, several starting structures have been chosen and the results with the lowest final energy are reported in this work. For the models ML3 and M3, a relaxed surface scan was performed first, where the dihedral angle $\varphi$ between the plane containing the $\mathrm{Al}^{3+}-\mathrm{N}-\mathrm{O}$ adsorption complex and the plane containing the atoms N, Al1, and Al2 was constrained to certain values, varied over a full circle in steps of $\Delta \varphi=10^{\circ}$. Here the functional B3LYP was chosen. For all atoms of the $\left[\mathrm{Al}_{3}\left(\mu_{3}-\mathrm{O}\right)(\mathrm{OOC})_{6}\right]^{+}$ group as well as for the $\mathrm{OH}^{-}, \mathrm{H}_{2} \mathrm{O}$, and $\mathrm{NO}$ ligands, the def2TZVP basis set was used again, whereas for all other atoms, the cheaper def2-SV $(\mathrm{P})^{67}$ basis set was chosen. The structures with $\varphi_{\min }$, where the energies approached their global minima, were chosen as the starting structures for the final geometry optimizations for both models ML3 and M3 which were performed twice on the B3LYP/def2-TZVP and PBE0/def2TZVP ${ }^{69-71}$ levels of theory. The latter has been proven to supply reliable energies as well as vibrational frequencies for the $\left[\mathrm{Al}_{3}\left(\mu_{3}-\mathrm{O}\right) \mathrm{F}(\mathrm{OOC})_{6}\right]$ building block in the presence of $\mathrm{H}_{2}$, $\mathrm{CO}, \mathrm{CO}_{2}$, or $\mathrm{H}_{2} \mathrm{O}$ ligands. ${ }^{72}$

Since DFT does not include dispersion forces, Grimme's atom-pairwise dispersion correction to the DFT energy with the Becke-Johnson damping ${ }^{73,74}$ was used in all calculations. More parameters of the DFT calculations are provided in the supplementary material. For the geometry optimized models
M1, M2, and M3, the vibrational frequencies were calculated numerically. For all three models, it turned out that those frequencies are all positive, verifying that the obtained structures are local energetic minima. Corresponding investigations of the Hessian matrices were not possible for the ML1, ML2, and ML3 models since here constrained optimizations were performed. But the similarity between the finally optimized MX and corresponding MLX (X = 1, 2, 3) structural models indicate that the latter are local energetic minima, too (see computational results section, Table S1 of the supplementary material, and Fig. 2).

Using the EPR/NMR module implemented in ORCA, the g-tensor, the electric field gradient at the $\mathrm{Al}$ and $\mathrm{N}$ sites, and the Fermi-contact and dipolar contributions to the hfi tensors of the ${ }^{27} \mathrm{Al}$ and ${ }^{1} \mathrm{H}$ nuclei next neighbored to the $\mathrm{NO}$ as well as the ${ }^{14} \mathrm{~N}$ nucleus of the NO molecule were calculated for the ML3 structure as optimized at the B3LYP/def2-TZVP and PBE0/def2TZVP levels. Several combinations of basis sets as well as functionals were examined. In all cases, the IGLO-III ${ }^{75}$ basis set was chosen for the next neighbored oxygens of all $\mathrm{Al}$ atoms as well as for the $\mathrm{NO}$ molecule and the oxygens of the $\mathrm{OH}^{-}$and $\mathrm{H}_{2} \mathrm{O}$ ligands. All other non-metallic atoms were treated with the EPR-II ${ }^{76}$ basis set. For the $\mathrm{Al}$ atoms, the different basis sets aug-cc-pVTZ, ${ }^{77}$ aug-cc-pVTZ-J, ${ }^{77-79}$ and IGLO-III were used in combination with the functionals B3LYP, PBE0, PBE, $, 0,81$

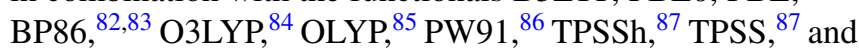
PWP $^{83,88,89}$ for calculations on the B3LYP optimized cluster ML3. The same functionals but only the Al basis set aug-ccpVTZ-J was used for the calculations on the PBE0 optimized cluster model ML3. In all cases, an accurate variant for the calculation of the spin-orbit-coupling operator was chosen with the keyword SOMF(1X). Exemplary calculations have shown that the orbital contribution to the hfi of the All atom is less than $0.05 \mathrm{MHz}$, justifying its negligence in the present contribution.

The binding energy ${ }^{72}$ was calculated using the counterpoise correction of the basis set superposition error as proposed by Boys and Bernardi. ${ }^{90}$ The analysis of the calculated wave functions was carried out with the aid of the program Chemissian version 4.43 .

\section{EXPERIMENTAL RESULTS}

\section{Field sweep experiments}

A 2-pulse ESE field sweep spectrum measured at the temperature $T=6 \mathrm{~K}$ with a pulse delay of $\tau=140 \mathrm{~ns}$ is presented in Fig. 3(a). It shows an EPR signal at field positions $340 \mathrm{mT}<B_{0}$ $<450 \mathrm{mT}$ with g-tensor principle values $g_{e}>g_{x x} \approx g_{y y}>g_{z z}$, which is typical for the ${ }^{2} \Pi_{1 / 2}$ ground state of $\mathrm{NO}$ adsorbed at some diamagnetic surface side. ${ }^{20} \mathrm{We}$ interpret the observed signal as the sum of three kinds of immobilized NO species called NOa, NOb, and NOc. Their simulations, shown in Fig. 3, are rough illustrations since the signal overlapping prevented a precise determination of all g-tensor principle values.

Species NOa has been already observed in a CW-EPR study published by Barth et al. ${ }^{25}$ In that publication, the signal of NOa was roughly simulated with g-tensor principle values slightly deviating from that shown in Table I. By CW-EPR, a value $g_{z z}=1.905(3)$ was determined ${ }^{25}$ for species NOa. 
a)

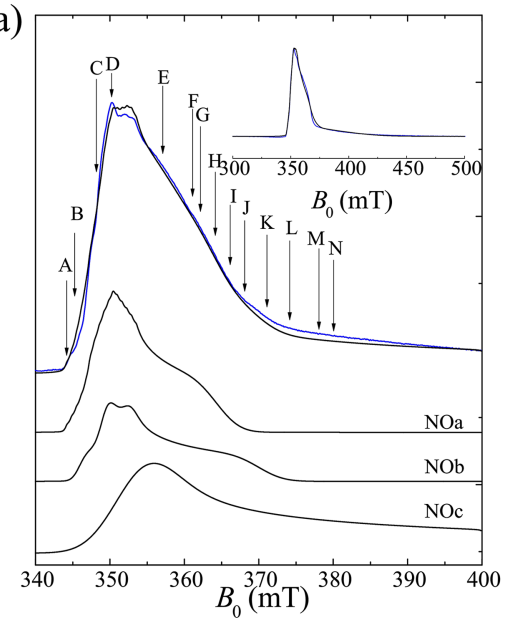

b)

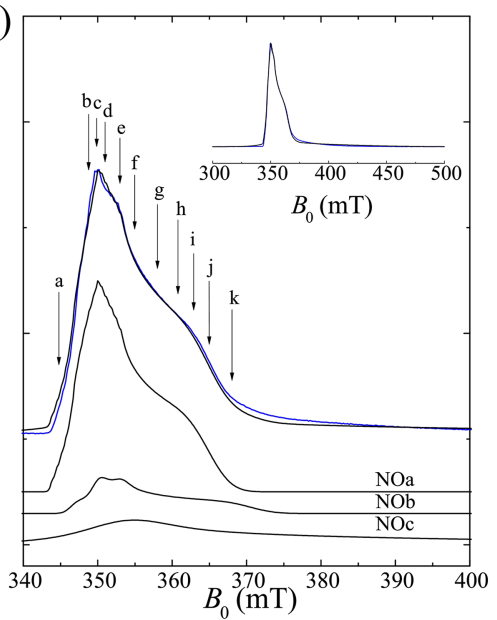

FIG. 3. 2-pulse ESE field sweep spectra (blue) measured at $T=6 \mathrm{~K}$ with non-selective pulses and an inter-pulse time delay $\tau=140 \mathrm{~ns}$ (a) and with selective pulses and an inter-pulse time delay $\tau=1200 \mathrm{~ns}$ (b). The insets show the corresponding spectra measured over a larger field range. Simulations of the NO adsorption species NOc, NOb, and NOa in MIL-100(Al) and their sum overlapping with the experimental spectrum are shown in black (from bottom to top). The arrows A to $\mathrm{N}$ label field positions, where HYSCORE experiments were conducted. The arrows a to $\mathrm{k}$ label the field positions for the different ENDOR experiments.
But in a 2-pulse ESE field sweep spectrum with longer interpulse delay $\tau$ [Fig. 3(b)], the signals of species NOb and NOc are suppressed, which might be explained by their shorter phase memory times. Thus, from this spectrum, a more precise determination of that parameter to $g_{z z}=1.909(3)$ is possible. Nevertheless, the ${ }^{14} \mathrm{~N}$ hfi parameter $A_{y y}^{14 \mathrm{~N}}$ determined by $\mathrm{CW}$ $\mathrm{EPR}^{25}$ equals the one we have determined in the present work (Table I) and also the strong hfi coupling to an ${ }^{27} \mathrm{Al}$ nucleus as observed in the work of Barth et al. ${ }^{25}$ is reproduced by the following results.

The 2-pulse ESE field sweep spectra show at high fields a stretched out tail of signal intensity up to field positions of about $B_{0}=470 \mathrm{mT}$ (see the insets in Fig. 3), indicating the presence of a large variety of weakly physisorbed $\mathrm{NO}$ species ${ }^{20}$ which we sum up with the label NOc. Its range of g-tensor principle values (Table I) has been roughly estimated by simulation (Fig. 3).

We attribute a pronounced spectral feature of the 2-pulse ESE field sweep spectrum with short inter-pulse delay $\tau$ at magnetic field positions $366 \mathrm{mT}<B_{0}<374 \mathrm{mT}$ to the $g_{z z^{-}}$ singularity of a third NO adsorption species NOb [Fig. 3(a)]. Its $g_{x x, y y}$ singularities are not resolved due to the overlapping with the signals of NOa and NOc. But the following analysis of the HYSCORE spectra measured at different magnetic fields leads to an estimation of all three principle values of its g-tensor within the errors given in Table I.

With the knowledge of the spin Hamiltonian parameters of species $\mathrm{NOa}, \mathrm{NOb}$ and $\mathrm{NOc}$, as derived from the pulsed EPR experiments in this work, a more precise simulation of the CW EPR signal of the considered sample at $T=8 \mathrm{~K}$, than that reported earlier, ${ }^{25}$ is possible as shown in Fig. 4. From the simulations of the CW EPR spectrum, considering all three $\mathrm{NO}$ adsorption species $\mathrm{NOa}, \mathrm{NOb}$, and NOc, their relative amounts could be estimated as given in Table I. For both species, $\mathrm{NOb}$ and NOc amounts are indicated, which are of the same order than that of species NOa. Nevertheless, the spectroscopic parameter of species NOb and NOc, as derived in the present work, do not allow the clear assignment to a structural model. Supportive $a b$ initio calculations have not resulted in reasonable results, yet, due to the lack of an appropriate guess of the starting structures for species NOb and NOc. Thus, the focus in the present work will be the characterization of species NOa, for which a structural model has been derived. The EPR signatures of species NOb and NOc, as they have been derived spectroscopically so far, are summarized in Tables I and II and are shortly discussed in the supplementary material.

\section{HYSCORE experiments}

The HYSCORE pulse sequence is based on the 3-pulse electron spin echo envelope modulation (ESEEM) sequence where the latter generates nuclear spin coherences that precess in the evolution period between the second and third pulses. ${ }^{59,60}$ The HYSCORE pulse schema introduces a $\pi$ mixing pulse in this evolution period that correlates nuclear frequencies in different electron spin submanifolds leading to characteristic cross peaks in the HYSCORE spectrum. Given any arbitrary nuclear spin $I$ coupled to an electron spin $S=1 / 2$, any nuclear frequency $v_{m_{I}, m_{I}^{\prime}}^{\alpha}$ of the $\alpha$-manifold can be correlated with any nuclear frequencies $v_{m_{I}^{\prime \prime}, m_{I}^{\prime \prime \prime}}^{\beta}$ of the $\beta$-manifold by such cross peaks, in principle. ${ }^{59}$ Those cross peaks occur in each quadrant with the frequency coordinates $\left( \pm v_{m_{I}, m_{I}^{\prime}}^{\alpha, \beta}, \pm v_{m_{I}^{\prime \prime}, m_{I}^{\prime \prime \prime}}^{\beta, \alpha}\right)$ and $\left( \pm v_{m_{I}, m_{I}^{\prime}}^{\alpha, \beta}, \mp v_{m_{I}^{\prime \prime}, m_{I}^{\prime \prime \prime}}^{\beta, \alpha}\right) .{ }^{91}$

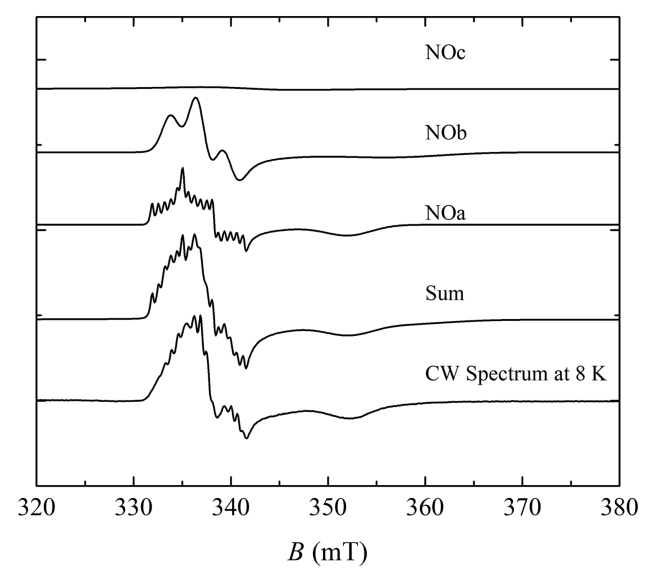

FIG. 4. CW-EPR spectrum measured at $8 \mathrm{~K}$ for the NO loaded sample MIL100(Al) (bottom, as published in Ref. 25) and the corresponding simulations of the NO adsorption species NOc, NOb, NOa, and their sum (from the top to bottom) with spin Hamilton parameters determined in this work (Tables I and II). Here species NOc has the same relative amount to species NOa as in Fig. 3(a). 
Due to the symmetry of the HYSCORE signal, it is sufficient to consider only cross peaks in the nonequivalent first and fourth quadrants with coordinates $\left(\left|v_{m_{I}, m_{I}^{\prime}}^{\alpha, \beta}\right|,\left|v_{m_{I}^{\prime \prime}, m_{I}^{\prime \prime \prime}}^{\beta, \alpha}\right|\right)$ and $\left(-\left|v_{m_{I}, m_{I}^{\prime}}^{\alpha, \beta}\right|,\left|v_{m_{I}^{\prime \prime}, m_{I}^{\prime \prime \prime}}^{\beta, \alpha}\right|\right)$, respectively.

In the HYSCORE spectra of powders, one observes ridges that consist of cross peaks $\left(v_{m_{I}, m_{I}^{\prime}}^{\alpha, \beta}(\Omega), v_{m_{I}^{\prime \prime}, m_{I}^{\prime \prime \prime}}^{\beta, \alpha}(\Omega)\right)$ depending on the orientation $\Omega$ between the molecular and laboratory frames. Since the phases of spin packets of different adjacent orientations might differ from each other, some ridges are suppressed by interference effects whereas other ridges are not. ${ }^{91}$ As a result, spin systems with small hfi values $|A|<2\left|v_{I}\right|$ show ridges that are dominant in the first quadrant and are suppressed in the fourth quadrant, whereas for strong hfi $|A|>2\left|v_{I}\right|$, the ridges are dominant in the fourth quadrant and are suppressed in the first. For intermediate couplings, ridges occur in both quadrants. ${ }^{59}$

To take advantage of the high resolution given by the unravelling of the nuclear spectra into two frequency dimensions, orientation selective ${ }^{59}$ HYSCORE spectra were measured at a temperature $T \approx 6 \mathrm{~K}$ and magnetic field positions A to $\mathrm{N}$ as indicated by arrows in Fig. 3(a). All spectra are shown in Figs. S2-S23 of the supplementary material. A representative spectrum measured at the magnetic field $B_{0}=350 \mathrm{mT}$ (position $\mathrm{C}$ ) is shown in Fig. 5. It shows a ridge (blue) which crosses the point $\left(\nu_{\mathrm{H}}, \nu_{\mathrm{H}}\right)$ with $\nu_{\mathrm{H}}=14.90 \mathrm{MHz}$ being the proton Lamor frequency and which is therefore indicative for distant proton species. This proton signal is visible in the HYSCORE spectra over the full magnetic field range covered by the observer positions A to N [Fig. 3(a), Figs. S2S23]. It shows no significant orientation selectivity indicating that several proton species contribute to this signal. Therefore an unambiguous determination of their hfi parameters is not possible. But from the maximum extension of the proton signals ridges, measured at the field positions $\mathrm{A}$ to $\mathrm{N}$, one can estimate, aided by simulations, a maximal proton hfi value of $A_{\max } \approx 3.7 \mathrm{MHz}$.

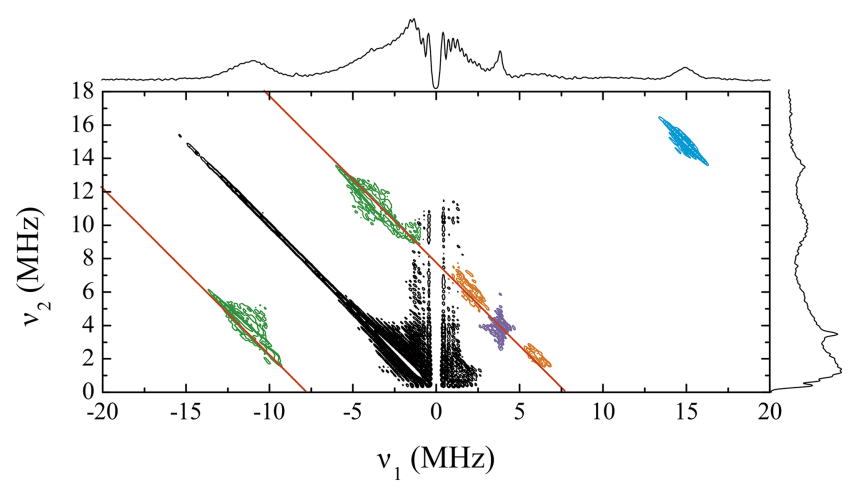

FIG. 5. HYSCORE spectrum measured at a magnetic field position $B_{0}=350 \mathrm{mT}$ at a temperature $T=6 \mathrm{~K}$. The spectrum shows the sum of two spectra measured with different time delays $\tau=92 \mathrm{~ns}$ and $\tau=128 \mathrm{~ns}$ to avoid the presence of blind spots for the aluminum and proton signals. The spectrum shows the presence of hyperfine couplings to three different ${ }^{27} \mathrm{Al}$ nuclear species called Ala (green), Alb (orange), and Alc (purple) as well as weak couplings to protons (blue). The black signals on the diagonal in the IV quadrant originate most probably from an incomplete inversion by the $\pi$-mixing pulse. ${ }^{59}$ The red lines indicate the ${ }^{27} \mathrm{Al}$ frequency anti-diagonals described by Eq. (5).
The HYSCORE spectrum in Fig. 5 shows also signals which extend along the diagonals

$$
v_{2}=-v_{1} \pm 2 v_{\mathrm{Al}},
$$

where $v_{\mathrm{Al}}=3.89 \mathrm{MHz}$ is the ${ }^{27} \mathrm{Al}$ nuclear Lamor frequency. Both diagonals are plotted as red lines in Fig. 5. Accordingly, we attribute these signals to three ${ }^{27} \mathrm{Al}$ nuclear species. ${ }^{92}$

One of those ${ }^{27} \mathrm{Al}$ species shows a signal in the fourth quadrant, which is indicative for the $\left(v_{-\frac{1}{2}, \frac{1}{2}}^{\alpha}, \nu_{-\frac{1}{2}, \frac{1}{2}}^{\beta}\right)$ ridge of an ${ }^{27} \mathrm{Al}$ species with large hf $|A|>2\left|v_{\mathrm{Al}}\right| \cdot .^{92}$ It is colored in green in Fig. 5. Its HYSCORE signals are visible at the field positions A to I but not J to N [see Fig. 3(a), Figs. S2-S23 of the supplementary material]. Even increasing the signal to noise ratio of the HYSCORE spectrum at the field position $\mathrm{J}$ by oversampling, ${ }^{59}$ setting $\Delta t_{1}=\Delta t_{2}=12 \mathrm{~ns}$, does not resolve any signal of Ala (Fig. S16 of the supplementary material). Consequently, we attribute this species to an ${ }^{27} \mathrm{Al}$ nucleus coupled to the $\mathrm{NO}$ adsorption species NOa, which we call here species Ala. Thorough simulations of orientation selective spectra, including also the ENDOR data which are presented below, allowed the determination of the full ${ }^{27} \mathrm{Al}$ hfi tensor of species Ala as given in Table II. In particular, the distinct orientation selective measurement of the Ala HYSCORE signals at the low and high magnetic field positions A and I allowed the determination of the hfi-tensors Euler angles $\alpha$ and $\beta$ (Figs. S2 and S15 of the supplementary material). The strong orientation selection at the observer position $\mathrm{A}$ is a consequence of the large ${ }^{14} \mathrm{~N}$ hfi splitting in the g-tensor principle y-direction, which shifts the $m_{I}=+1$ EPR transition to such low fields that hardly any other orientation contributes to the EPR signal as it is illustrated in Fig. S24 of the supplementary material. Two exemplary spectra measured at the magnetic field position $\mathrm{C}$ with different $\tau$ values are shown together with the simulated Ala signals in Fig. 6. Note the $\tau$ dependent intensity modulations of the proton and Ala signals (Fig. S1 of the supplementary material). All simulations are collected in the supplementary material (Figs. S2-S15). Both the significant distribution of the Ala HYSCORE signal intensity along the diagonals described by Eq. (5) and the broad linewidths of the Ala ENDOR signals, which are presented below, were taken into account by a linewidth model, where the parameter $a_{\text {iso }}^{\text {Ala }}$ was assumed to be Gaussian distributed with a standard deviation $\Delta a_{\text {iso }}^{\mathrm{Ala}}=2 \mathrm{MHz}$. All HYSCORE simulations also include Gaussian distributions of the ${ }^{14} \mathrm{~N}$ hfi parameters $A_{x x}^{14 \mathrm{~N}}$ and $A_{z z}^{14 \mathrm{~N}}$ with standard deviations $\Delta A_{x x}^{14 \mathrm{~N}}=\Delta A_{z z}^{14 \mathrm{~N}}=1.25 \mathrm{MHz}$. They model the linewidths of the ${ }^{14} \mathrm{~N}$ ENDOR signals as discussed below but only have minor influence on the simulated Ala HYSCORE signals. The ${ }^{14} \mathrm{~N}$ nqi was set to zero as it is not resolved in the experimental spectra. In addition, for all HYSCORE simulations, the excitation band width was set to a quite large value of $\Delta v=62.5 \mathrm{MHz}$ to account for the experimental inhomogeneous broadening which was not reproduced by the assumed distribution model for the parameters $a_{\text {iso }}^{\mathrm{Ala}}, A_{x x}^{14 \mathrm{~N}}$, and $A_{z z}^{14 \mathrm{~N}}$ alone.

Interestingly we do not observe other ridges, correlating single quantum (SQ) transitions, than those comprising the central transition $\left(v_{-\frac{1}{2}, \frac{1}{2}}^{\alpha}, v_{-\frac{1}{2}, \frac{1}{2}}^{\beta}\right)$ cross peaks although the 

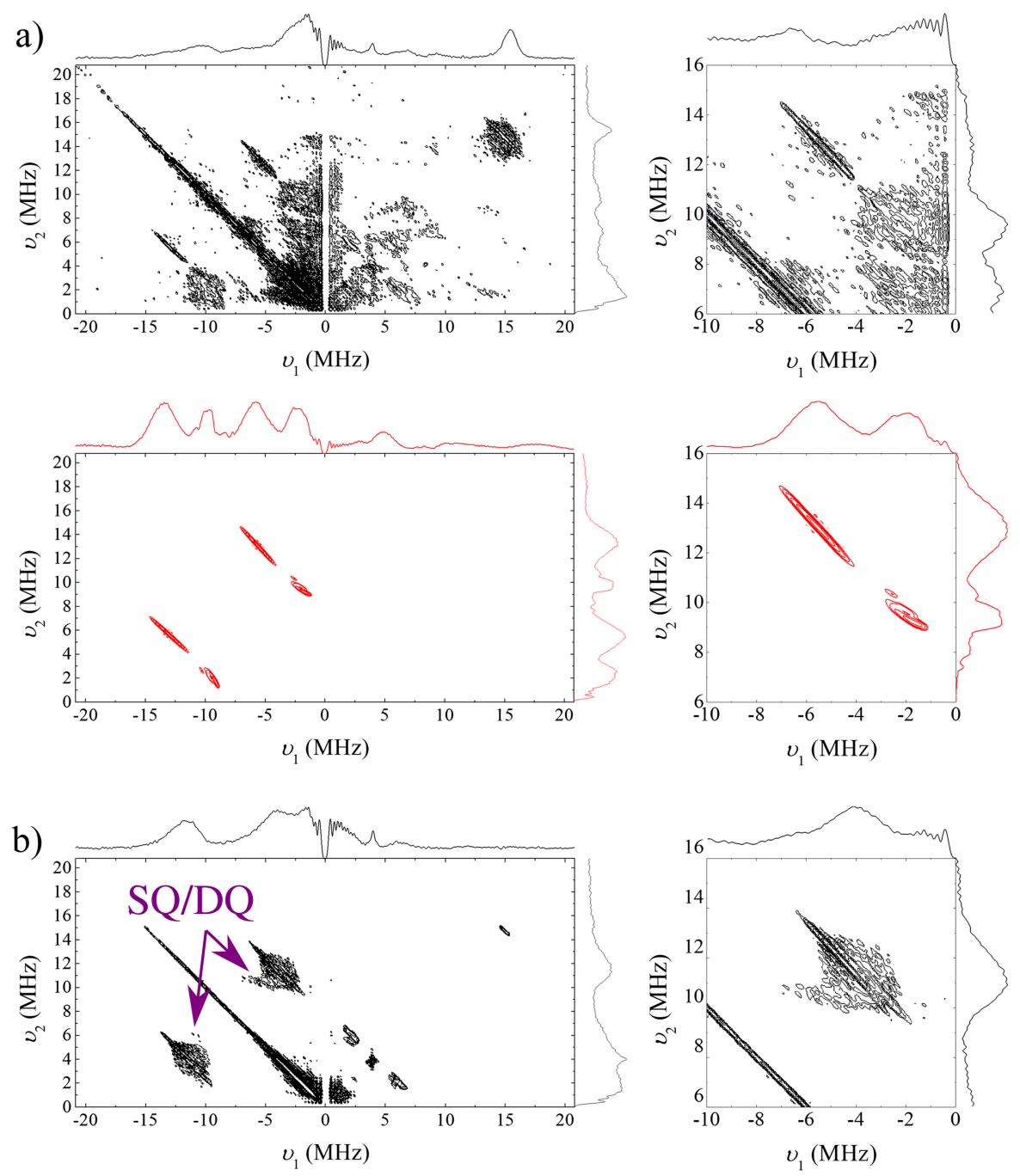

FIG. 6. Experimental (black) and simulated Ala (red) HYSCORE spectra at $T=6 \mathrm{~K}$ and $B_{0}=348.0 \mathrm{mT}$ with $\tau=92$ ns (a) and $\tau=130 \mathrm{~ns}$ (b). Enlargements of the left spectra are shown on the right.
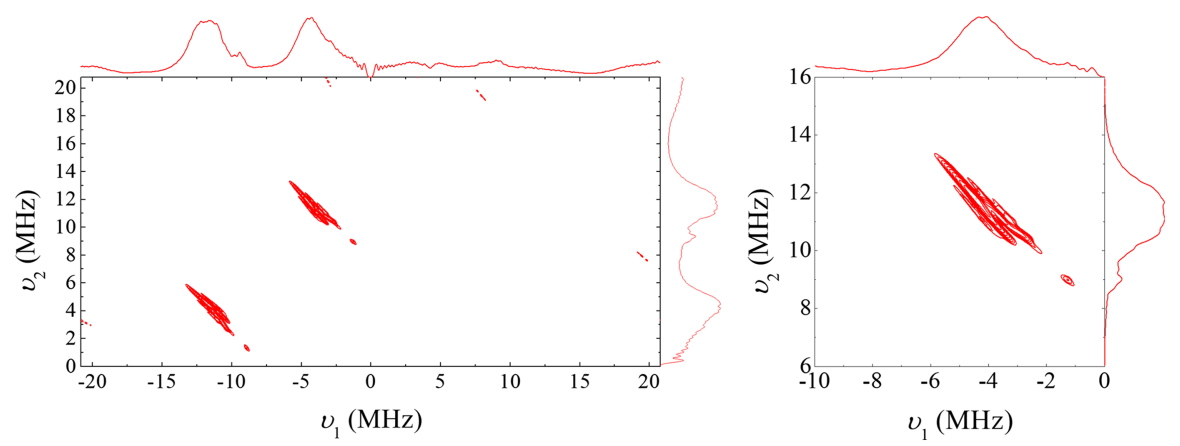

${ }^{27} \mathrm{Al}$ nucleus has a nuclear spin $I=5 / 2$. We explain the almost complete absence of other HYSCORE signals than the $\left(v_{-\frac{1}{2}, \frac{1}{2}}^{\alpha}, v_{-\frac{1}{2}, \frac{1}{2}}^{\beta}\right)$ ridge for species Ala by their first order nqi broadening [Eq. (4)]. ${ }^{92}$ Since the $\left(v_{-\frac{1}{2}, \frac{1}{2}}^{\alpha}, v_{-\frac{1}{2}, \frac{1}{2}}^{\beta}\right)$ ridge depends on the nqi only in second order, ${ }^{59}$ we have set the nqi to zero in the simulations, as we also did for the simulation of the ENDOR spectra. Exemplary simulations, using a MIL-100 ${ }^{27} \mathrm{Al}$ nqi reported in the literature, ${ }^{8}$ have justified this simplification (Figs. S25-S27 of the supplementary material). Nevertheless, we observe in addition to the $\left(v_{-\frac{1}{2}, \frac{1}{2}}^{\alpha}, v_{-\frac{1}{2}, \frac{1}{2}}^{\beta}\right)$ ridge of species Ala broad and less intense signals in certain spectra, which we attribute to correlations between SQ and double quantum (DQ) transitions and which are indicated by arrows in Fig. 6(b).

The HYSCORE signal of the second species Alb, which is colored in orange in Fig. 5, occurs in the first quadrant, indicating weak hfi couplings $|A|<2\left|v_{\mathrm{Al}}\right| . .^{59}$ It can be observed at the magnetic field positions $\mathrm{B}$ to $\mathrm{J}$ but not $\mathrm{A}$ and $\mathrm{K}$ to $\mathrm{N}$ (see the supplementary material). Thus, we attribute this signal to an ${ }^{27} \mathrm{Al}$ species coupled to the $\mathrm{NO}$ adsorption species NOb. Its spin Hamiltonian parameters have been roughly determined as collected in Tables I and II. Since the characterization of species NOb is not the content of the present investigation, we will refer for more information to the supplementary material. 
a)

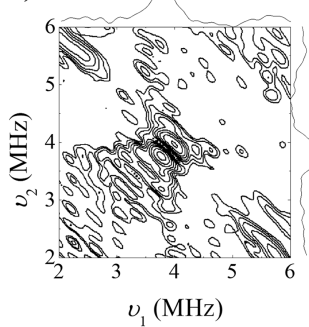

b)

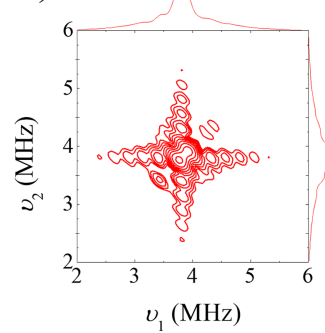

c)

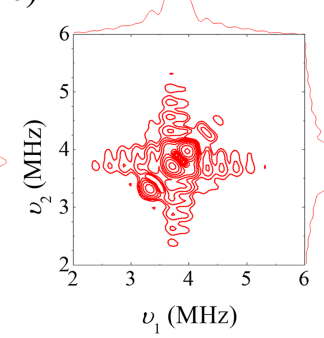

FIG. 7. Experimental (a) and simulated Alc (b), (c) HYSCORE spectra at $T=6 \mathrm{~K}$ and $B_{0}=348.0 \mathrm{mT}$ applying an inter-pulse delay $\tau=92 \mathrm{~ns}$. Simulations with different nqi coupling parameters (b) $C_{Q}=4.5 \mathrm{MHz}$, $\eta=0.26$ and (c) $C_{Q}=6.0 \mathrm{MHz}, \eta=0.26$ are presented.
The third resolved ${ }^{27} \mathrm{Al}$ species Alc has its HYSCORE signal (purple in Fig. 5) also in the first quadrant, but it hardly extends beyond the spectral region of the ${ }^{27} \mathrm{Al}$ Lamor frequency cross peak $\left(v_{\mathrm{Al}}, v_{\mathrm{Al}}\right)$. We therefore attribute this species to the distant matrix ${ }^{27} \mathrm{Al}$ nuclei. Its signals are observable at all magnetic field positions A to $\mathrm{N}$. Therefore, one can conclude that at least $\mathrm{NO}$ species NOc interacts with distant ${ }^{27} \mathrm{Al}$ nuclei.

HYSCORE spectra of species Alc measured at different magnetic fields are shown in Fig. S28 of the supplementary material. They show almost no orientation selection, indicating that a variety of ${ }^{27} \mathrm{Al}$ nuclei contribute to those signals, as one would expect for distant matrix nuclei. Almost all Alc HYSCORE signals show two maxima, one slightly above and one slightly below the ${ }^{27} \mathrm{Al}$ nuclear Lamor frequency, as it is shown in Fig. 7(a) for an exemplary spectrum measured at $B_{0}=348.0 \mathrm{mT}$ (see also Fig. S28). We have simulated those signals with an equally distributed sum of powder spectra summed up over all Euler angles describing the relative orientation between the ${ }^{27} \mathrm{Al} \mathrm{hfi}$ and nqi tensors (Fig. 7, Fig. S28). For that, we have switched off any orientation selection according to the spherical-averaging approximation. ${ }^{63}$ No distribution in the spin-Hamiltonian parameters was considered for the simulations presented in Fig. 7(b) and Fig. S28. For them, we have set $C_{Q}=4.5 \mathrm{MHz}, \eta=0.26 \mathrm{MHz}$, $a_{\text {iso }}=0 \mathrm{MHz}$, and $T=0.05 \mathrm{MHz}$. Those parameters are within the simulation derived error ranges listed in Table II. The determined nqi parameters of species Alc are comparable to those measured by NMR spectroscopy for ${ }^{27} \mathrm{Al}$ nuclei in the MIL-100(Al) material. ${ }^{8}$ Thus we attribute species Alc to distant Al nuclei at the regular metal sites of MIL-100(Al). From the upper bound of $T$, one can estimate, within a pointdipole approximation, ${ }^{59}$ that the matrix ${ }^{27} \mathrm{Al}$ nuclei have minimal distances to the NO molecule of $r=4.1 \AA$. A simulation based analysis of the orientation dependency of the corresponding ENDOR frequencies shows that the two maxima that occur directly below and above the ${ }^{27} \mathrm{Al}$ Lamor frequency (Fig. 7) are the $\theta_{\text {nqi }}=40.4^{\circ}$ and $\theta_{\text {nqi }}=90^{\circ}$ powder edge singularities of the $\left(v_{-\frac{1}{2}, \frac{1}{2}}^{\alpha}, v_{-\frac{1}{2}, \frac{1}{2}}^{\beta}\right)$ powder spectrum, where $\theta_{\text {nqi }}$ is the angle between the external magnetic field and the principle z-direction of the nqi tensor. Whereas the low frequency maximum could be fitted with a value $C_{Q}=4.5 \mathrm{MHz}$, a larger value $C_{Q}=6.0 \mathrm{MHz}$ was necessary to fit the high frequency maximum, as it is shown in Fig. 7(c). This indicates a significant distribution of at least the parameter $C_{Q}$, which is governed by the simulation derived range given in Table II and which might reflect the structural disorder in the MIL-100(Al) $\mathrm{MOF}^{26}$ as already indicated by NMR spectroscopy. ${ }^{8}$

\section{Davies ENDOR experiments}

Orientation selective ${ }^{59}$ ENDOR spectra were measured at a temperature $T=6 \mathrm{~K}$ at field positions indicated by arrows in Fig. 3(b) and are shown in Figs. 8 and 9. We attribute the signals symmetrically distributed around the proton Lamor frequency $v_{\mathrm{H}} \approx 15 \mathrm{MHz}$ to the $v_{\alpha, \beta}^{\mathrm{H}}=v_{\mathrm{H}} \pm \frac{A}{2}$ spectral components of weakly coupled proton species. ${ }^{59}$ The proton signals show almost no orientation selectivity comparing the spectra measured at the field positions a to j (Fig. 8), indicating
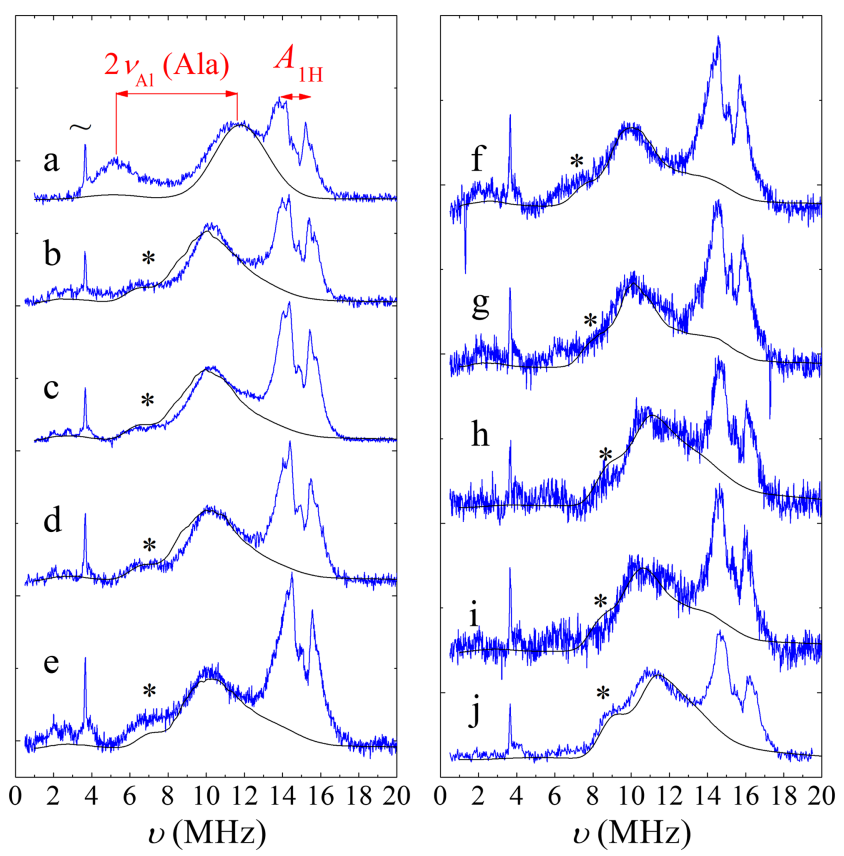

FIG. 8. Davies ENDOR spectra (blue) measured at $T=6 \mathrm{~K}$ at magnetic field positions $B_{0}=345.0 \mathrm{mT}$ (a), $348.7 \mathrm{mT}$ (b), $349.8 \mathrm{mT}$ (c), $350.7 \mathrm{mT}$ (d), 352.8 $\mathrm{mT}(\mathrm{e}), 355.3 \mathrm{mT}(\mathrm{f}), 358.0 \mathrm{mT}(\mathrm{g}), 360.7 \mathrm{mT}(\mathrm{h}), 362.9 \mathrm{mT}$ (i), and 365.0 $\mathrm{mT}(\mathrm{j})$. The letters a ... j denote the corresponding field positions indicated by arrows in Fig. 3(b), where the orientation selective ENDOR spectra have been recorded. Simulations of the NO adsorption species NOa coupled to the ${ }^{27} \mathrm{Al}$ nucleus Ala are shown in black. Here an mw excitation width of $\Delta v_{\mathrm{mw}}$ $=60 \mathrm{MHz}$ as well as an isotropic convolutional Voigtian broadening with a full width at half maximum (FWHM) of $\Delta v=0.16 \mathrm{MHz}$ was assumed to account for the inhomogeneous broadened line shape. The Ala nqi was set to zero in all simulations for the reasons explained in the text. The symbols and $*$ indicate an experimental artefact signal, whose position does not change with the magnetic field and signals of the NO ${ }^{14} \mathrm{~N}$ nitrogen, respectively. The splitting of the ${ }^{27} \mathrm{Al}$ doublet of the strongly coupled Ala species and of the doublet of weakly coupled protons is labeled in red. 
a)

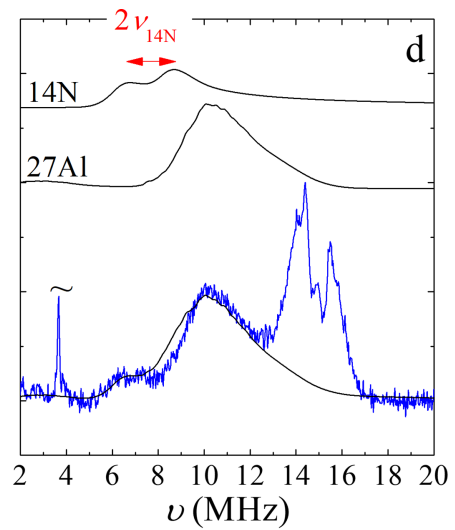

b)

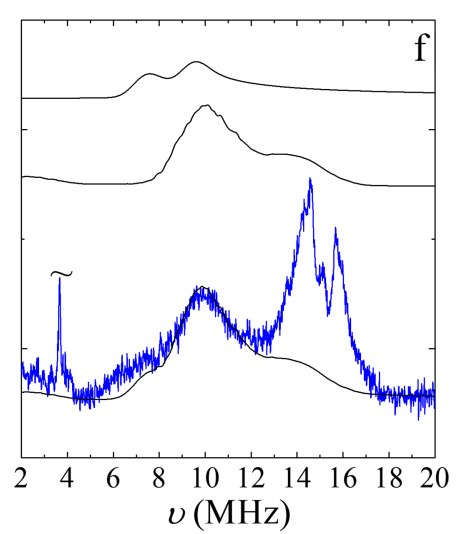

C)

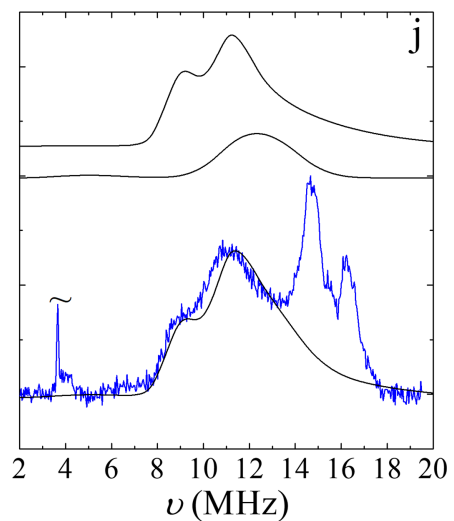

d)

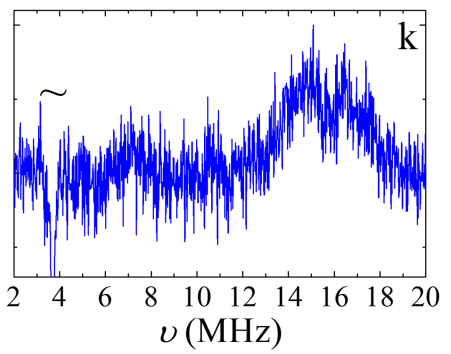

FIG. 9. Selected experimental Davies ENDOR spectra (blue) measured at $T=6 \mathrm{~K}$ at magnetic field positions $B_{0}=350.7 \mathrm{mT}$ (a), $355.3 \mathrm{mT} \mathrm{(b),} 365.0 \mathrm{mT}$ (c), and $368.0 \mathrm{mT}$ (d). The latin letters at the top right label the magnetic field positions as indicated by arrows in Fig. 3(b). The upper two signals (black), shown in (a)-(c), are the simulated ENDOR signals of the NO ${ }^{14} \mathrm{~N}$ nucleus and the $\mathrm{Ala}{ }^{27} \mathrm{Al}$ nucleus coupled to the $\mathrm{NO}$ adsorption species NOa. Their sums are shown in black below overlapping with the experimental spectra. The splitting of the ${ }^{14} \mathrm{~N}$ doublet of the simulated signal is labeled in red. The symbol $\sim$ denotes an artefact signal. that a variety of different proton species contribute to them. The widths of the proton ENDOR signals allow an estimation $A_{\max } \approx 3.5 \mathrm{MHz}$ for the maximal hfi of the proton signals at field positions a to $\mathrm{j} .{ }^{59}$ Thus we attribute those signals to the same proton species as we have observed by HYSCORE spectroscopy. Interestingly, the proton signal measured at the field position $\mathrm{k}\left[B_{0}=368.0 \mathrm{mT}\right.$, Fig. $\left.9(\mathrm{~d})\right]$ provides a larger value of $A_{\max } \approx 5.0 \mathrm{MHz}$, which might have been not resolved by the HYSCORE experiments at corresponding field positions due to the low signal intensity. At these field positions, species $\mathrm{NOb}$ or NOc are expected to contribute most significantly to the EPR spectrum in contrast to species NOa [Fig. 3(b)], indicating differences in the variety of neighbored proton species among the different NO adsorption moieties.

In addition, ENDOR spectra measured at magnetic fields $B_{0}<368 \mathrm{mT}$ show a broad signal at frequencies of $v \approx 7.7 \ldots 17.3 \mathrm{MHz}$, which partially overlaps with the proton signals (Fig. 8). This signal can be attributed to the frequencies $v^{\mathrm{Al}} \approx \frac{A}{2}+v_{\mathrm{Al}}$ of the Ala ENDOR signal of the $m_{S}=+1 / 2$ manifold since it can be simulated by the same model as used for the simulations of the corresponding HYSCORE signals. As verified by an exemplary simulation (Fig. S27 of the supplementary material), an ${ }^{27} \mathrm{Al}$ nqi, as reported for the regular $\mathrm{Al}$ species in MIL-100(Al), ${ }^{8}$ is not expected to be resolved due to the large linewidth of the ENDOR signal originating from distributions of the nqi parameters and the isotropic hfi parameter $a_{\mathrm{iso}}^{\mathrm{Ala}}$. The weak intensity of the frequencies $v^{\mathrm{Al}} \approx \frac{A}{2}-v_{\mathrm{Al}}$ of the ENDOR signals belonging to the $m_{S}=-1 / 2$ manifold, as observed at almost all field positions, can be explained by the hyperfine enhancement effect, which increases the ENDOR signal intensity at high nuclear frequencies to a larger extent than at low frequencies. ${ }^{59,93}$ This is verified by the simulations shown in Fig. 8 which include the hyperfine enhancement effect. Only at the lowest observer field position a, the low frequency part of the Ala ENDOR signal seems to be more intense in the experimental spectrum than it is reproduced by the simulation (Fig. 8).

At the field position $\mathrm{k}\left(B_{0}=368 \mathrm{mT}\right)$, where only the NO adsorption species $\mathrm{NOb}$ and NOc contribute to the electron spin echo, no signal of species Ala was measured anymore [Fig. 9(d)] which confirms its attribution to species NOa.

In most spectra, a less intense shoulder joins the Ala $v_{\alpha}^{\mathrm{Al}} \approx \frac{A}{2}+v_{\mathrm{Al}}$ spectral component at its low frequency end, which is indicated in Fig. 8 by the symbol $*$ and which could not be explained by the nuclear species Ala alone. It was tentatively assigned to the ENDOR signals of the $\mathrm{NO}{ }^{14} \mathrm{~N}$ nucleus. Since we do not observe any signal of ${ }^{14} \mathrm{~N}$ at the field position $\mathrm{k}\left[B_{0}=368 \mathrm{mT}\right.$, see Fig. 9(d)], where only signals of species $\mathrm{NOb}$ and NOc contribute to the 2-pulse ESE field sweep spectrum [Fig. 3(b)], we attribute the observed ${ }^{14} \mathrm{~N}$ signal to the $\mathrm{NO}$ adsorption species NOa. At the magnetic field positions $\mathrm{d}\left(B_{0}=350.7 \mathrm{mT}\right)$ and $\mathrm{j}\left(B_{0}=365.0 \mathrm{mT}\right)$, mainly orientations either lying in the NOa g-tensor $\mathrm{x}, \mathrm{y}$-plane or pointing parallel to the NOa g-tensor principle z-direction are excited. Since the ${ }^{14} \mathrm{~N}$ hfi tensor is known to be almost coaligned with the g-tensor, ${ }^{94}$ one can roughly estimate from the corresponding ENDOR spectra measured at those field positions [Figs. $9(\mathrm{a})$ and $9(\mathrm{c})]$ the principle values $A_{x x}^{14 \mathrm{~N}}$ and $A_{z z}^{14 \mathrm{~N}}$, respectively, as they are summarized in Table I. Here we interpret the resolved ${ }^{14} \mathrm{~N}$ ENDOR signals belonging to the frequencies $v_{\alpha}^{\mathrm{N}} \approx\left|\frac{A_{x x, z z}}{2}+v_{14 \mathrm{~N}}\right|$ of the $m_{S}=+1 / 2$ manifold, with $A_{x x, z z}<0$ 
TABLE III. Spin Hamilton parameters as calculated on the B3LYP and PBE0 levels with the Al atoms aug-ccpVTZ, aug-cc-pVTZ-J, and IGLO-III basis sets for the model cluster ML3 finally optimized using the B3LYP functional. Values determined experimentally in the present work are also given. The g-tensor Euler angles $\alpha^{g}$, $\ldots, \gamma^{g}$ refer to a molecular frame, whose $\mathrm{z}$-axis is defined to be parallel to the internuclear axis of the NO molecule and whose $\mathrm{x}$-axis is perpendicular to the plane spanned by the NO molecule and the All atom [Fig. 11(c)]. The indices Al1 and $\mathrm{N}$ designate parameters related to the All and N atoms, whereas the indices hfi and nqi label Euler angles of the corresponding hfi or nqi interaction tensors.

\begin{tabular}{|c|c|c|c|c|c|}
\hline NO & $\begin{array}{c}\text { B3LYP/ } \\
\text { aug-cc-pVTZ }\end{array}$ & $\begin{array}{c}\text { PBE0/ } \\
\text { aug-cc-pVTZ }\end{array}$ & $\begin{array}{c}\text { B3LYP/ } \\
\text { aug-cc-pVTZ-J }\end{array}$ & $\begin{array}{l}\text { B3LYP/ } \\
\text { IGLO-III }\end{array}$ & Expt. \\
\hline$g_{x x}$ & 2.0062 & 2.0062 & 2.0062 & 2.0063 & $1.997(3)^{\mathrm{a}}$ \\
\hline$g_{y y}$ & 2.0023 & 2.0023 & 2.0023 & 2.0023 & $1.997(3)^{\mathrm{a}}$ \\
\hline$g_{z z}$ & 1.9519 & 1.9479 & 1.9518 & 1.9517 & $1.909(3)$ \\
\hline$\alpha^{g}(\operatorname{deg})$ & 89.76 & 89.74 & 90.07 & 90.00 & $\ldots$ \\
\hline$\beta^{g}(\operatorname{deg})$ & 2.24 & 2.29 & 2.19 & 2.20 & $\ldots$ \\
\hline$\gamma^{g}(\mathrm{deg})$ & -89.28 & -89.24 & -89.63 & -89.58 & $\ldots$ \\
\hline$a_{\mathrm{iso}}^{\mathrm{All}}(\mathrm{MHz})$ & 21.67 & 22.82 & 23.81 & 23.71 & $14.7(17)$ \\
\hline$T^{\mathrm{All}}(\mathrm{MHz})$ & 3.59 & 3.55 & 3.61 & 3.62 & $3.4(5)$ \\
\hline$\rho^{\mathrm{Al} 11}$ & 0.029 & 0.026 & 0.030 & 0.030 & $<0.5$ \\
\hline $\boldsymbol{\alpha}_{\mathrm{hfi}}^{\mathrm{Al1}}(\mathrm{deg})$ & 89.22 & 89.20 & 89.24 & 89.28 & $90(30)$ \\
\hline$\beta_{\mathrm{hfi}}^{\mathrm{All}}(\mathrm{deg})$ & 47.95 & 47.71 & 47.96 & 47.97 & $50(10)$ \\
\hline$\gamma_{\mathrm{hfi}}^{\mathrm{All}}(\mathrm{deg})$ & 89.71 & 89.73 & 89.22 & 89.43 & $\ldots$ \\
\hline$C_{Q}^{\text {All }}(\mathrm{MHz})$ & 4.28 & 4.04 & 2.80 & 2.96 & $\ldots^{\mathrm{b}}$ \\
\hline$\eta^{\text {All }}$ & 0.326 & 0.329 & 0.104 & 0.20 & $\ldots{ }^{b}$ \\
\hline $\boldsymbol{\alpha}_{\mathrm{nqi}}^{\mathrm{Al1}}(\mathrm{deg})$ & 85.06 & 85.54 & 83.83 & 93.96 & $\ldots^{\mathrm{b}}$ \\
\hline$\beta_{\mathrm{nqi}}^{\mathrm{Al} 1}(\mathrm{deg})$ & 73.00 & 73.52 & 90.14 & 29.40 & $\ldots^{\mathrm{b}}$ \\
\hline$\gamma_{\mathrm{nqi}}^{\mathrm{All}}(\mathrm{deg})$ & 85.77 & 86.04 & 101.53 & 84.63 & $\ldots^{\mathrm{b}}$ \\
\hline$A_{x x}^{\mathrm{N}}(\mathrm{MHz})$ & -17.92 & -19.95 & -17.86 & -17.80 & $\pm 14(8)^{\mathrm{c}}$ \\
\hline$A_{y y}^{\mathrm{N}}(\mathrm{MHz})$ & 86.38 & 82.47 & 86.42 & 86.52 & $\pm 86(3)^{\mathrm{a}, \mathrm{c}}$ \\
\hline$A_{z z}^{\mathrm{N}}(\mathrm{MHz})$ & -18.27 & -20.80 & -18.22 & -18.17 & $\pm 20(6)^{\mathrm{c}}$ \\
\hline$a_{\mathrm{iso}}^{\mathrm{N}}(\mathrm{MHz})$ & 16.73 & 13.91 & 16.78 & 16.85 & $17.3(57)^{d}$ \\
\hline$T^{\mathrm{N}}(\mathrm{MHz})$ & 34.83 & 34.28 & 34.82 & 34.84 & $34.3(34)^{\mathrm{d}}$ \\
\hline$\rho^{\mathrm{N}}$ & -0.01 & -0.01 & -0.01 & -0.01 & $0.5(10)^{d}$ \\
\hline$\alpha_{\mathrm{hfi}}^{\mathrm{N}}(\mathrm{deg})$ & 69.98 & 81.68 & 76.26 & 72.99 & $0^{\mathrm{e}}$ \\
\hline$\beta_{\mathrm{hfi}}^{\mathrm{N}}(\operatorname{deg})$ & 0.78 & 0.90 & 0.81 & 0.80 & $0^{\mathrm{e}}$ \\
\hline$\gamma_{\mathrm{hfi}}^{\mathrm{N}}(\mathrm{deg})$ & -70.23 & -81.90 & -76.44 & -73.16 & $0^{\mathrm{e}}$ \\
\hline$C_{Q}^{\mathrm{N}}(\mathrm{MHz})$ & 6.84 & 6.67 & 6.84 & 6.84 & $\ldots{ }^{b}$ \\
\hline$\eta^{\tilde{N}}$ & 0.398 & 0.375 & 0.398 & 0.398 & $\ldots{ }^{b}$ \\
\hline$\alpha_{\mathrm{nqi}}^{\mathrm{N}}(\mathrm{deg})$ & 179.77 & -0.21 & -0.17 & -0.15 & $\ldots{ }^{b}$ \\
\hline$\beta_{\mathrm{nqi}}^{\mathrm{N}}(\operatorname{deg})$ & 90.03 & 89.97 & 89.98 & 89.98 & $\ldots^{\mathrm{b}}$ \\
\hline$\gamma_{\mathrm{nqi}}^{\mathrm{N}}(\mathrm{deg})$ & 90.10 & 89.07 & -89.83 & 89.90 & $\ldots^{\mathrm{b}}$ \\
\hline
\end{tabular}

${ }^{\mathrm{a}}$ Data from the work of Barth et al. ${ }^{25}$ which are confirmed by the present work.

${ }^{\mathrm{b}}$ Not resolved.

${ }^{\mathrm{c}}$ Only the magnitude but not the sign was experimentally derived.

${ }^{\mathrm{d}}$ Assuming signs of the ${ }^{14} \mathrm{~N}$ hi principle values as calculated by DFT.

${ }^{\mathrm{e}}$ Values are not rigorously experimentally derived but are typically for $\mathrm{NO}$ adsorption species. ${ }^{94}$

according to the DFT derived results (Table III) ${ }^{59}$ and with $v_{14 \mathrm{~N}}$ being the ${ }^{14} \mathrm{~N}$ nuclear Lamor frequency. We suppose that the larger frequencies $v_{\beta}^{\mathrm{N}} \approx\left|\frac{A_{x x, z z}}{2}-v_{14 \mathrm{~N}}\right|$ of the $m_{S}=-1 / 2$ manifold component overlap with the Ala ENDOR signal. This assumption is rationalized by no resolved second ${ }^{14} \mathrm{~N}$ signal in any ENDOR spectrum, which should be present with significant intensity as it is indicated by the spectral simulations (see Fig. 9).

Note that the ENDOR spectral simulations did not allow for the determination of the absolute or relative signs of the ${ }^{14} \mathrm{~N}$ hfi principle values. We suppose that this can be rationalized by the large pseudo-secular ${ }^{14} \mathrm{~N}$ hfi coupling, which dominates qualitatively the orientation dependency of the ENDOR frequencies. ${ }^{59}$ Furthermore, the experimental ENDOR signals did not resolve any ${ }^{14} \mathrm{~N}$ nqi. Thus we set this interaction to zero in the respective simulations. The effect of an eventual ${ }^{14} \mathrm{~N}$ nqi in the order of the DFT derived values (Table III) on the experimental determination of the ${ }^{14} \mathrm{~N}$ hfi parameters has been considered by the corresponding errors given for the latter (Table III). 


\section{COMPUTATIONAL RESULTS}

For the DFT calculation, one has to choose the exchangecorrelation functional that partly includes electron correlation in an approximate fashion. Unfortunately there exists no systematic approach improving the approximation of the exchange-correlation. ${ }^{95}$ Thus one has to verify the applicability of a functional with either bench mark calculations or the experiment. Applying the first approach for a single $\mathrm{Al}^{3+}$ ion penta-coordinated to five oxygens and various small molecules adsorbed at the remaining axial CUS, Mavrandonakis et al. ${ }^{72}$ has shown that the PBE0/def2-TZVP and B3LYP/def2-TZVP levels of theory including the dispersion correction scheme of Grimme $^{74}$ show a good performance for the calculation of the interaction energies. Thus we have used both approaches for the present geometry optimizations.

Files containing the coordinates of all finally optimized cluster models are provided in the supplementary material. Some structural parameters of the models M1, ML1, M2, and ML2 are given in Table S1 of the supplementary material.

A part of the potential energy surface (PES) of the models M3 and ML3 was calculated varying the dihedral angle $\varphi$ between the plane containing the $\mathrm{Al}^{3+}-\mathrm{N}-\mathrm{O}$ adsorption complex and the plane containing the atoms $\mathrm{N}, \mathrm{Al1}$, and $\mathrm{Al} 2$ as explained in the Computational methods section. For an angle $\varphi=0^{\circ}$, the NO molecule is tilted towards the $\mathrm{Al} 2-\mathrm{H}_{2} \mathrm{O}$ site, whereas for $\varphi=180^{\circ}$, the NO molecule is tilted towards the Al3-OH site. The calculated single point energies are shown in Fig. 10. For both models, they have their global minima at orientations where $\varphi \approx 180^{\circ}$, namely, at angles $\varphi=170^{\circ} \pm 10^{\circ}$
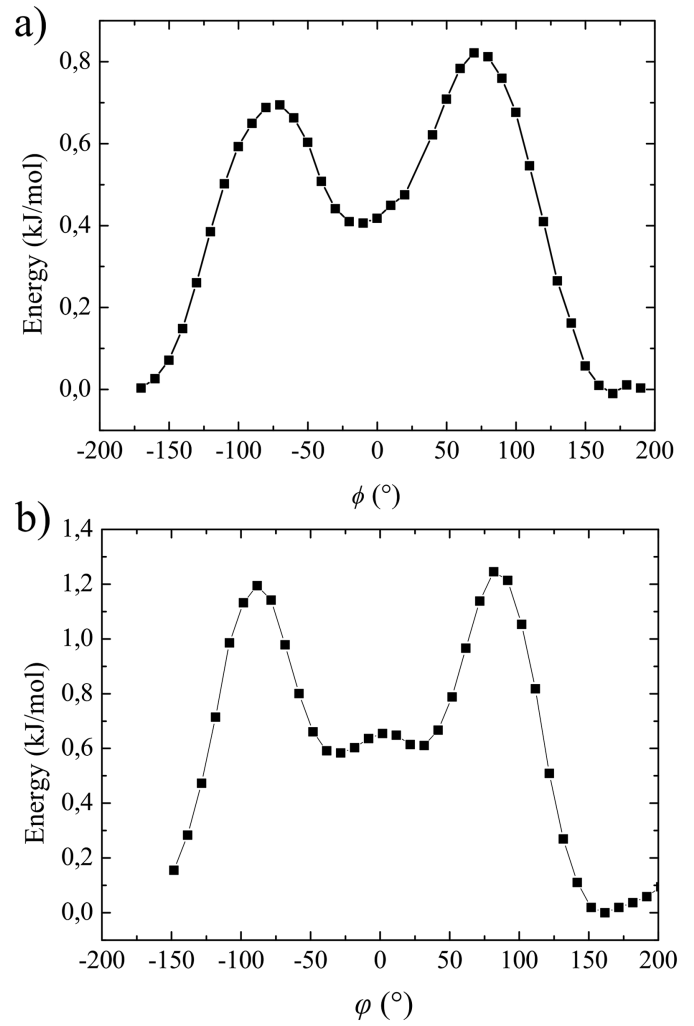

FIG. 10. Relative single point energies of the cluster models ML3 (a) and M3 (b) calculated during relaxed surface scans where the dihedral angle was varied.
TABLE IV. Structural parameters describing the NO adsorption complex at the A11 CUS of the cluster models ML3 and M3 as optimized with the B3LYP and PBE0 functionals. The length $r_{\mathrm{Al}-\mathrm{NO}}$ is the distance between the $\mathrm{N}$ and the Al1 atoms, the parameter $\theta_{\mathrm{Al}-\mathrm{N}-\mathrm{O}}$ is the Al1-N-O bond angle, and $\varphi_{A-\mathrm{AlNO}}$ is the angle between the plane A containing all aluminum atoms and the plane containing the NO molecule and the atom Al1.

\begin{tabular}{lccc}
\hline \hline & $r_{\mathrm{Al}-\mathrm{NO}}(\AA)$ & $\theta_{\mathrm{Al}-\mathrm{N}-\mathrm{O}}(\mathrm{deg})$ & $\varphi_{\mathrm{A}-\mathrm{AlNO}}(\mathrm{deg})$ \\
\hline ML3/B3LYP & 2.460 & 127.71 & 7.14 \\
ML3/PBE0 & 2.374 & 128.37 & 5.81 \\
M3/B3LYP & 2.654 & 126.11 & 20.65 \\
M3/PBE0 & 2.550 & 126.95 & 22.22 \\
\hline \hline
\end{tabular}

and $\varphi=162^{\circ} \pm 10^{\circ}$ in the case of the models ML3 and M3, respectively. These global minima were the starting structures for further geometry optimizations, where $\varphi$ was allowed to fully relax. Structural parameters describing the NO adsorption complex and pictures of the finally optimized cluster models ML3 and M3 are given in Table IV and Fig. 2, respectively. Additional structural parameters characterizing the $\mathrm{H}_{2} \mathrm{O}$ and $\mathrm{OH}^{-}$adsorption complexes for all cluster models are listed in Table $\mathrm{S} 1$ of the supplementary material.

The Al-NO adsorption complex of cluster model ML3 has a bent end-on structure where the NO molecule is aligned almost parallel to the plane A containing all three $\mathrm{Al}$ atoms and is tilted towards the hydroxyl group by an angle $\theta_{\mathrm{Al}-\mathrm{N}-\mathrm{O}} \approx 128^{\circ}$ (Fig. 2, Table IV). The calculated binding energy of the NO molecule at the Al site of model ML3 at $T=0 \mathrm{~K}$ is $\Delta E_{\mathrm{NO}}=-22.4 \mathrm{~kJ} / \mathrm{mol}$.

It is known that the exact prediction of isotropic hfi parameters by DFT is difficult due to eventual inaccuracies in the calculation of spin polarization. ${ }^{96}$ As a consequence, the performance of different functionals for the calculation of the hfi is discussed controversially in the literature. For example, Hermosilla et al. ${ }^{97}$ reported a large variation of the calculated isotropic hfi with the chosen DFT methods for the secondand third-row nuclei among various organic and inorganic radicals. For the ${ }^{27} \mathrm{Al}$ nucleus of the $\mathrm{Al}_{2}{ }^{-}$radical electronic ground state, they found good agreement between the experimental isotropic hfi and the B3LYP/TZVP results. Saladino and Larsen ${ }^{98}$ reported a better performance of half-and-half hybrid functionals than pure generalized gradient correction (GGA) functionals for the calculation of the isotropic hfi of the ${ }^{51} \mathrm{~V}$ nucleus in $\mathrm{VO}^{2+}$ complexes. On the other hand, Sundararajan and Neese ${ }^{99}$ reported good agreement of the ${ }^{14} \mathrm{~N}$ isotropic hfi of NO-bound myoglobin with the experiment for GGA functionals, whereas the hybrid functionals B3LYP and PBE0 underestimated this parameter. To account this unpredictability of choosing the right functional for the prediction of hfi parameters by DFT, we calculated the ${ }^{27} \mathrm{Al}$ and ${ }^{14} \mathrm{~N}$ hfi of the Al1 and N atoms of the B3LYP/def2-TZVP and PBE0/def2TZVP optimized clusters ML3 with various GGA functionals (BP86, OLYP, PBE, PW91, PWP), hybrid functionals (PBE0, B3LYP, O3LYP), and the meta-GGA functional TPSS as well as its hybrid version TPSSh (Table III, Tables S2 and S3 of the supplementary material). All GGA functionals give large values for $a_{\text {iso }}^{\mathrm{All}}$ but small values for $a_{\mathrm{iso}}^{14 \mathrm{~N}}$. The B3LYP, PBE0, and TPSSh functionals give the smallest values for $a_{\text {iso }}^{\mathrm{All}}$ and $T^{\mathrm{Al1}}$ and large values for $a_{\text {iso }}^{14 \mathrm{~N}}$, indicating that the inclusion of 
a)

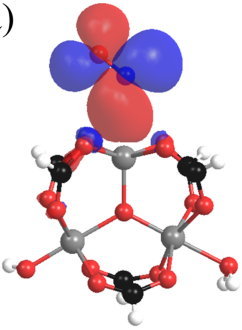

c)

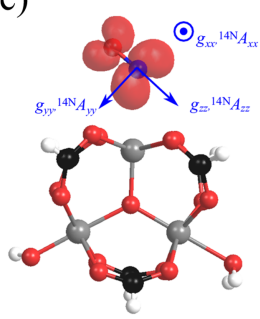

b)

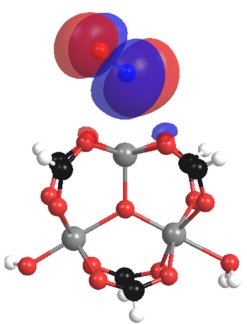

d)

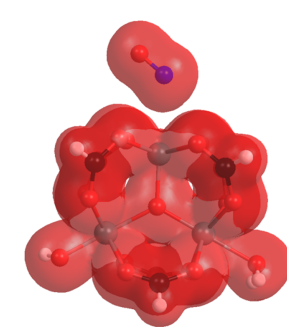

FIG. 11. The antibonding $\pi_{y}^{*}$ HOMO containing the unpaired electron (a), the antibonding $\pi_{x}^{*}$ LUMO (b), the spin density (c), and the electronic density (d) as calculated at the B3LYP/def2-TZVP level for the model cluster M3. The atoms are colored in white $(\mathrm{H})$, black $(\mathrm{C})$, blue $(\mathrm{N})$, red $(\mathrm{O})$, and gray $(\mathrm{Al})$. The principle directions of the NOa g-tensor and ${ }^{14} \mathrm{~N}$ hfi-tensor are illustrated in (c) in blue.

exact exchange leads to a larger localization of the spin-density at the $\mathrm{N}$ atom than at the Al1 atom. The PBE0 optimized structure has systematically larger Al1 hfi coupling parameters than the B3LYP optimized cluster which is probably related to its $0.1 \AA$ shorter distance $r_{\mathrm{Al}-\mathrm{NO}}$ between the All and $\mathrm{N}$ atoms (Table IV).

The orientations of all hfi tensors were calculated relatively to the g-tensor. The latter, as calculated with the B3LYP, PBE0, and TPSSh functionals for the B3LYP optimized structure $\mathbf{M L 3}$, has principle values $g_{x x} \gtrsim g_{y y} \approx g_{e}>g_{z z}$, where $g_{e}$ is the free electron g-value (Table III, Table S2 of the supplementary material). Its $\mathrm{z}$-axis is almost parallel to the $\mathrm{N}-\mathrm{O}$ bond axis, and its $\mathrm{x}$-axis is perpendicular to the plane containing the NO molecule and the All atom. Note that the y-axis is oriented along the $\pi_{y}^{*} \mathrm{MO}$ [Fig. 11(c)].

More magnetic parameters, calculated at the B3LYP, PBE0, and TPSSh levels for the B3LYP optimized clusters ML2 and ML3 with different Al basis sets, are summarized in Table III and Tables S2-S6 of the supplementary material, including the hfi and nqi tensors of the Al1, Al2, Al3, and $\mathrm{N}$ atoms and the hit tensors of the next neighbored protons of the four btc ligands directly connected to the CUS where NO binds. Consistently, the ${ }^{14} \mathrm{~N}$ hfi tensor is coaligned with the g-tensor, as it is typically for the adsorbed NO molecule [Fig. 11(c)]. ${ }^{94}$

\section{DISCUSSION}

By distinct pulse EPR experiments on samples of MIL100(Al), we have observed three NO adsorption species NOa, $\mathrm{NOb}$, and NOc, where the first one interacts with a strongly coupled ${ }^{27} \mathrm{Al}$ nucleus Ala, the second one interacts with a weakly coupled ${ }^{27} \mathrm{Al}$ nucleus $\mathrm{Alb}$, and at least, species NOc is indicated to interact with the distant matrix ${ }^{27} \mathrm{Al}$ nuclei Alc of the MIL-100(Al) framework. The signals of weakly coupled protons have been also observed by ENDOR and HYSCORE spectroscopy at all observer field positions.

In this section, we want to discuss the experimental results regarding the NO adsorption species NOa because a structural model was found for this species in this work. This goal has not been achieved for the species NOb and NOc. Nevertheless, the small g-tensor values of NOc indicate small electric surface fields at its adsorption site. Thus an adsorption of NOc on the organic part of the MIL-100(Al) material seems most likely. Further results concerning those two species are discussed in the supplementary material.

We have determined by DFT geometry optimization the zero temperature ground state of an $\mathrm{NO}$ adsorption complex at the $\mathrm{Al}^{3+}$ CUS of a representative cluster model ML3, cut from the MIL-100(Al) crystal structure. Almost all B3LYP derived ${ }^{14} \mathrm{~N}$ and ${ }^{27} \mathrm{Al}$ hfi parameters coincide with the experimental parameters (Table III). Only the computed ${ }^{27} \mathrm{Al}$ isotropic hfi constant $a_{\mathrm{iso}}^{\mathrm{All}}$ is about $7 \mathrm{MHz}$ larger than the experimental one, which corresponds to about $0.2 \%$ spin density difference in the $\mathrm{Al} 3 \mathrm{~s}$ valence orbital. ${ }^{100}$ Difficulties in the accurate calculation of small spin density and spin polarization might indeed lead to significant deviations of the calculated isotropic hfi parameters from the actual values, ${ }^{96,97}$ which is also illustrated by the large variation of the calculated isotropic ${ }^{27} \mathrm{Al} \mathrm{hfi} \mathrm{with}$ varying functionals in the present work (Table $\mathrm{S} 3$ of the supplementary material), correlating with the included amount of exact exchange. On the other hand, the measured anisotropic ${ }^{27} \mathrm{Al} \mathrm{hfi}$ and ${ }^{14} \mathrm{~N}$ parts, which should reflect the geometry of the complex more precisely than the isotropic hfi parts, equals the B3LYP derived ones with good accuracy (Table III). Thus we suggest that an NO molecule adsorbed at a CUS of the MIL-100(Al) MOF is the most likely interpretation for the observed EPR active species NOa. In particular, the present work indicates that among the various functionals applied here, the B3LYP functional shows the best performance for the calculation of the geometric and electronic structure of the model ML3 since it reproduces the EPR derived hfi parameters most accurately.

It is noteworthy that the two other aluminum nuclei $\mathrm{Al} 2$ and $\mathrm{Al} 3$ of the optimized model cluster ML3 have distances to the NO molecule of about $r_{\mathrm{Al}-\mathrm{NO}} \approx 5.4 \AA$. Consequently their DFT derived isotropic hfi parameters are almost zero and their calculated dipolar hfi coupling parameters are $T \approx 0.13 \mathrm{MHz}$ (Table $\mathrm{S} 4$ of the supplementary material). Thus their EPR signals should only contribute to those of distant matrix aluminum nuclei Alc (Table II), which also implies that $\mathrm{Al} 2$ or $\mathrm{Al} 3$ cannot be attributed to the observed aluminum species Alb interacting with NOa.

The four nearest neighbored protons of the NO molecule from the four adjacent btc ligands in the optimized cluster model ML3 have distances $r_{\mathrm{H}-\mathrm{NO}} \approx 4.0 \AA$ A to the NO molecule and consequently their DFT derived hfi parameters are $a_{\text {iso }} \approx 0 \mathrm{MHz}$ and $T \approx 1.1 \mathrm{MHz}$ (Table S5 of the supplementary material). Thus their ENDOR and HYSCORE signals might contribute to that variety of weakly coupled proton signals observed experimentally.

The B3LYP derived NO adsorption complex of model ML3 has a bent end-on structure with an $\mathrm{Al}-\mathrm{N}$ distance $r_{\mathrm{Al}-\mathrm{NO}}=2.46 \AA$ and an $\mathrm{Al}-\mathrm{N}-\mathrm{O}$ bond angle $\theta_{\mathrm{Al}-\mathrm{N}-\mathrm{O}} \approx 128^{\circ}$ 
(Table IV). Similar parameters $r_{\mathrm{Na}-\mathrm{NO}}=2.1 \AA$ and $\theta_{\mathrm{Na}-\mathrm{N}-\mathrm{O}}$ $\approx 142^{\circ}$ have been roughly derived for an $\mathrm{Na}^{+}-\mathrm{NO}$ adsorption complex in zeolite $\mathrm{Na}-\mathrm{A}$ from detailed ENDOR data. ${ }^{15}$ This supports the accuracy of the calculated ML3 geometry since the $\mathrm{Na}^{+}$ion has the same electron configuration and a similar size as the $\mathrm{Al}^{3+}$ ion. The somewhat larger distance $r_{\mathrm{Al}-\mathrm{NO}}$ might be related to a partial shielding of the electric surface field at the $\mathrm{Al}^{3+}$ CUS by the four nearest neighbored planar oxygen atoms.

The Mulliken and Löwdin atomic charges and spin populations of the atom Al1 and the NO molecule are similar for the optimized models ML3 and M3 (Tables S7 and S8 of the supplementary material), as also their geometric properties are (Table IV). This indicates that the electronic structure of the NO adsorption complex mainly depends on the metal-oxygen cluster, whereas the btc ligands have only minor influence on its properties.

The $\pi_{y}^{*}$ highest occupied canonical molecular orbital (HOMO) involved in the NO binding and containing the unpaired electron, the $\pi_{x}^{*}$ lowest unoccupied canonical molecular orbital (LUMO), the spin density, and the electronic density of the model M3 are shown in Fig. 11. The corresponding MOs and densities are qualitatively the same for the optimized model ML3. As indicated by those pictures, the HOMO, which determines the main characteristics of the bonding, ${ }^{101}$ contains almost all spin density. The Mulliken and Löwdin spin population analysis of the B3LYP/def2-TZVP derived model structure ML3 deviate somewhat (Table S7 of the supplementary material), but the trend is clear: About 95\%-97\% of the spin density is located at the NO molecule, only $2 \%-4 \%$ on the Al1 atom and less than $0.5 \%$ on each of the four next neighbored planar oxygen atoms. A spin density of about 53\%-62\% is in the $2 \mathrm{p}_{\mathrm{y}}$ orbital of the NO nitrogen atom, whereas somewhat less spin density of $34 \%-36 \%$ populates the $2 \mathrm{p}_{\mathrm{y}}$ orbital of the NO oxygen atom. The experimentally determined ${ }^{14} \mathrm{~N}$ dipolar hfi constant is $T^{N}=34.3 \mathrm{MHz}$ (Table III), assuming the DFT derived signs for the ${ }^{14} \mathrm{~N}$ hfi principle values (Table III). This translates to about $61 \%$ spin density in the $2 p_{y}$ atomic orbital (AO) at the nitrogen atom ${ }^{100}$ which is in good agreement with the DFT derived spin density populations (Table S7). The experimentally determined ${ }^{14} \mathrm{~N}$ isotropic hfi constant is $a_{i s o}^{\mathrm{N}}=17.3 \mathrm{MHz}$ (Table III) if one assumes the DFT derived signs of the ${ }^{14} \mathrm{~N}$ hfi principle values (Table III). This indicates that about $1.0 \%$ spin density occupies the $2 \mathrm{~s} \mathrm{AO}$ of the ${ }^{14} \mathrm{~N}$ atom. ${ }^{100}$ This value is almost equal to the Löwdin population analysis for the B3LYP/def2-TZVP optimized model ML3 (Table S7). The Mulliken population analysis predicts a value of $-0.02 \%$, indicating the better performance of the Löwdin compared to the Mulliken method. ${ }^{95}$ This different accuracy of both approaches is also confirmed by the predicted spin densities in the Al1 s-type AOs (Table S7). According to Löwdin, about $0.21 \%$ spin density occupies the Al1 AOs with s-character which is in good agreement with the value $0.38 \%$ as derived from the experimentally determined isotropic ${ }^{27} \mathrm{Al}$ hfi value $a_{i s o}^{\mathrm{All}}=14.7 \mathrm{MHz}$ (Table II). ${ }^{100}$ Here the Mulliken analysis seems to fail since it predicts a small negative spin density in the s-type AOs of the Al1 atom (Table S7).

The previous population analysis reflects the nature of the NO $\pi_{y}^{*}$ orbital, which is understood qualitatively in the first approximation as a linear combination of the two $2 \mathrm{p}_{\mathrm{y}}$ AOs of the NO nitrogen and oxygen atoms. ${ }^{16}$ Indeed, an analysis of the composition of the $\pi_{y}^{*}$ HOMO in terms of contributing AOs according to Mulliken mirrors the spin density population. Especially no distinct s, p, or d orbitals of the Al1 or next neighbored oxygen atom contribute with more than $2 \%$ to the HOMO. Consistently, the electronic density has small values in between the NO and All atoms, as shown in Fig. 11(d). Therefore this NO adsorption complex lacks any covalent bonding character which is also reflected in a calculated Mayer bond order of about 0.13 for the N-Al1 bond. The Mayer bond orders for the bonds between the NO nitrogen and the planar oxygens are even smaller than 0.01. Thus, the present wave function analysis suggest an almost purely ionic interaction between the NO and the MIL-100(Al) surface at the CUS without significant covalency, as one would expect for a bond between NO and the closed shell cation $\mathrm{Al}^{3+} \cdot 11$

A weak ionic binding is also reflected by the small calculated binding energy $\Delta E_{\mathrm{NO}}=-22.4 \mathrm{~kJ} / \mathrm{mol}$ of the NO molecule in the model cluster ML3 assuming zero temperature, which is more typical for physisorption rather than chemisorption. ${ }^{11}$ A similar binding energy of $\Delta E_{\mathrm{CO}}=-28.1 \mathrm{~kJ} / \mathrm{mol}$ was calculated for the $\mathrm{CO}$ molecule bonded to an $\mathrm{Al}^{3+} \mathrm{CUS}$ of an analogous $\mu_{3}$-oxo-bridged cluster model. ${ }^{72}$ This NO binding energy is still much larger than the energy barrier $\Delta E_{\mathrm{PES}}$ $\approx 0.8 \mathrm{~kJ} / \mathrm{mol}$ defined by the part of the calculated PES at zero temperature, which was scanned with respect to the dihedral angle $\varphi$ between the plane containing the $\mathrm{Al}^{3+}-\mathrm{N}-\mathrm{O}$ adsorption complex and the plane containing the atoms $\mathrm{N}, \mathrm{Al1}$, and Al2 [Fig. 10(a)]. Thus the DFT derived energies indicate that the NO molecule adsorbed at the MIL-100(Al) CUS is able to rotate almost freely around the $\mathrm{Al}-\mathrm{N}$ bond axis at intermediate temperatures, where it still binds to this metal site. This might be related to the occurrence of a second NO adsorption species at temperatures $T>20 \mathrm{~K}$ as reported elsewhere. ${ }^{25}$ Its larger g-value $g_{z z}=1.947(3)$ might indicate a motional averaging of the g-tensor principle values like it has been observed for a thermally activated two-site jump process for a $\mathrm{Na}^{+}-\mathrm{NO}$ complex in the NaA zeolite. ${ }^{94}$ The increase of the linewidth with rising temperatures as observed by $\mathrm{CW}$ EPR in the temperature range $8 \mathrm{~K}<T<160 \mathrm{~K}^{25}$ might be also related to this rotational motion.

We have also calculated by DFT a wavenumber $\bar{v}=2002 \mathrm{~cm}^{-1}$ for the $\mathrm{N}-\mathrm{O}$ stretching vibration of the optimized model cluster M3. This value is larger than $\bar{v}=1876 \mathrm{~cm}^{-1}$ of gaseous $\mathrm{NO}^{102}$ which might refer to the small depopulation of the antibonding $\pi_{y}^{*}$ HOMO due to a small but distinct transfer of electron density to the Al1 atom. But interestingly no IR signal of NO adsorbed in MIL-100(Al), which could be distinctly attributed to the $\mathrm{NO}-\mathrm{Al}^{3+}$ species, was observed in an earlier work down to the temperature $T=173 \mathrm{~K} .{ }^{25}$ We cannot explain this observation for sure and suggest therefore additional IR experiments at lower temperatures since the species NOa might have already been desorbed at $T=173 \mathrm{~K}$, even on the IR time scale. Nevertheless, it remains unclear why IR signals of NO, weakly physisorbed at the organic ligands, are still observable at that temperature. ${ }^{25}$ However our results indicate that the interaction of the $\mathrm{NO}$ molecule to the $\mathrm{Al}^{3+}$ 
CUS is only weak, even at zero temperature. It seems reasonable that at $T=173 \mathrm{~K}$ the IR signal of the NO- $\mathrm{Al}^{3+}$ moieties might contribute to the large variety of IR signals around $\bar{v}$ $=1876 \mathrm{~cm}^{-1}$ which were attributed to weakly physisorbed NO. ${ }^{25}$ An additional large inhomogeneous broadening of that IR signal might have also hindered its distinct detection. Such a distribution of the IR frequencies is indicated by the calculated flat PES (Fig. 10) leading eventually to a large structural variation of the adsorption complex at the CUS or might be related to the general structural disorder of the MOF. ${ }^{26}$

The electric surface field at the NO adsorption site can be characterized by the g-tensor principle values (Table I). From them, one can estimate in second order the energy difference $\Delta$ between the NO ${ }^{2} \Pi_{y}$ molecular state, where the unpaired electron resides in the $\pi_{y}^{*} \mathrm{MO}$, and the excited ${ }^{2} \Pi_{x}$ molecular state, where the unpaired electron resides in the $\pi_{x}^{*}$ MO. ${ }^{94}$ Thus, the energy $\Delta$ corresponds to the energy difference between the canonical HOMO and LUMO introduced above. ${ }^{94,103}$ It is a measure of the strength of the non-axial component of the electric field gradient at the adsorption site. ${ }^{20}$ If this component is zero, $\Delta=0$, and the ${ }^{2} \Pi_{1 / 2}$ state is degenerated, whereas $\Delta$ increases with the magnitude of the electric field gradient. Table V collects the g-tensor derived parameter $\Delta$ for the NO adsorption species NOa (this work), for the NO adsorption species at an extra-framework aluminum site in the H-ZSM-5 zeolite, here called species NOd, ${ }^{11}$ and for $\mathrm{NO}-\mathrm{Na}^{+}$adsorption complexes in the two zeolites NaZSM-5 (NOe) and Na-A (NOf). ${ }^{104}$ Obviously those values are of the same order, indicating that the electric surface fields are similar for all those complexes. Especially the values for NOa and NOd are almost the same. Note that the DFT derived HOMO-LUMO gap (Table V) is much too large compared to the experimental value. This might be related to the overestimation of the g-tensor principle values by DFT. The calculated $g_{x x}$ value is physically meaningless since it is larger than the free electron g-value $g_{e}=2.0023$ (Table III). Assuming that $g_{x x}$ $=2.0023$, one derives in second order ${ }^{94}$ from the DFT derived g-tensor principle values (Table III) a value $\Delta \approx 330 \mathrm{~kJ} / \mathrm{mol}$ that nicely fits to the overestimated DFT derived HOMO-LUMO gap (Table V). Therefore, the second order calculation of the gtensor principle values seems to be still a good approximation for the present system and the failure of DFT for the calculation

TABLE V. Energy splitting, $\Delta$, as calculated from the g-tensor principle values of the NO adsorption species according to the formulas given in the work of Rudolf et al. ${ }^{94}$ and the ${ }^{14} \mathrm{~N}$ hfi parameter $A_{y y}^{14 \mathrm{~N}}$ for various NO adsorption species. For species NOb and NOc, $\Delta$ is listed in Table $\mathrm{S} 9$ of the supplementary material. The HOMO-LUMO gap, calculated at the B3LYP/def2-TZVP level for the optimized model ML3, is also given.

\begin{tabular}{lccc}
\hline \hline Species & $T(\mathrm{~K})$ & $\Delta(\mathrm{kJ} / \mathrm{mol})$ & $A_{y y}^{14 \mathrm{~N}}(\mathrm{MHz})$ \\
\hline NOa [MIL-100(Al)] & 6 & $23.3(73)$ & $86(3)$ \\
NOd (H-ZSM-5 $\left.{ }^{\mathrm{a}, \mathrm{b}}\right)$ & 10 & 25.5 & $84.1(62)$ \\
NOe (Na-ZSM-a $\left.{ }^{\mathrm{a}, \mathrm{c}}\right)$ & 10 & $15.9(28)$ & $102.0(62)$ \\
NOf (Na-A $\left.{ }^{\mathrm{a}, \mathrm{c}}\right)$ & 10 & $26.2(38)$ & $91.6(62)$ \\
B3LYP/def2-TZVP (ML3) & 0 & 317.5 & $\ldots$ \\
\hline \hline
\end{tabular}

${ }^{\mathrm{a}} \mathrm{NO}$ adsorbed on zeolite matrix.

${ }^{\mathrm{b}} \mathrm{g}$-tensor values from the work of Rudolf et al., ${ }^{11}$ and our calculated value $\Delta$ differs from that stated there.

${ }^{\mathrm{c}}$ Rudolf et al. ${ }^{104}$ of the correct g-tensor principle values seems to originate from a wrong calculation of the excited ${ }^{2} \Pi_{x}$ molecular state energy of the NO molecule. Indeed a correct description of an excited state by DFT is problematic if this state has the same spatial and spin symmetry as the ground state has, ${ }^{95}$ like it is the case for the ${ }^{2} \Pi_{x}$ excited state of the present system. For such cases, the orthogonality between the excited and ground states is difficult to ensure by DFT. ${ }^{95}$ Nevertheless, the calculated orientation of the g-tensor matches the theoretically predicted: ${ }^{20}$ The smallest principle value points along the internuclear bond of the NO molecule, and the intermediate value points almost along the symmetry axis of the $2 \mathrm{p}_{\mathrm{y}}$ AOs contributing to the $\pi_{y}^{*}$ $\mathrm{HOMO}$ at the NO [Fig. 11(c)].

The experimental determined ${ }^{14} \mathrm{~N}$ hfi parameter $A_{y y}^{14 \mathrm{~N}}$ is a quantitative measure for the Lewis acidity of the $\mathrm{Al}^{3+} \mathrm{CUS}$ of the MIL-100 material. ${ }^{11}$ The electronic structure of the NO molecule can be interpreted as a mixture of two resonance structures

$$
: \dot{\mathrm{N}}=\ddot{\mathrm{O}}: \leftrightarrow: \ddot{\mathrm{N}}^{-}=\dot{\mathrm{O}}^{+},
$$

where most of the unpaired electron density is either in the $2 \mathrm{p}_{\mathrm{y}} \mathrm{AO}$ at the nitrogen or in the $2 \mathrm{p}_{\mathrm{y}} \mathrm{AO}$ at the oxygen atoms, respectively. ${ }^{11,16,105}$ The electron pair acceptor property of a Lewis acid site leads to a redistribution of the electron density in terms of the contributions of both resonance structures to the total electronic state of the NO molecule. The stronger the Lewis acidity of the adsorption site, the more unpaired electron density is shifted from the nitrogens $2 \mathrm{p}_{\mathrm{y}} \mathrm{AO}$ to the oxygen $2 \mathrm{p}_{\mathrm{y}} \mathrm{AO}$ in favor for an increased contribution of an additional electron pair at the nitrogen to the total electronic state. This redistribution of the electron density is measured by the ${ }^{14} \mathrm{~N}$ hfi parameter $A_{y y}^{14 \mathrm{~N}}$ since the latter is proportional to the amount of unpaired spin density in the nitrogen $2 \mathrm{p}_{\mathrm{y}}$ AO. ${ }^{100}$ According to this hfi parameter, the Lewis acidity of the MIL-100(Al) CUS seems similar to that of the extra-framework Al site in the H-ZSM-5 zeolite probed by species NOd (Table V). ${ }^{11}$ It has been claimed that acetonitrile $\left(\mathrm{CD}_{3} \mathrm{CN}\right)$ is a good IR spectroscopic probe to characterize the Lewis acidity of such sites. ${ }^{22}$ More specific the Lewis acidity can be probed by its $v(\mathrm{CN})$ wavenumber. For the $\mathrm{CD}_{3} \mathrm{CN}$ adsorbed at the MIL-100(Al) CUS, $v(\mathrm{CN})$ lies in the range $2326 \mathrm{~cm}^{-1}-2321 \mathrm{~cm}^{-1} .22$ For $\mathrm{CD}_{3} \mathrm{CN}$ adsorbed at the Al-Lewis acid site in H-ZSM-5 zeolite, IR spectroscopy provides a similar wavenumber $v(\mathrm{CN})$ $=2322 \mathrm{~cm}^{-1}$, ${ }^{106}$ confirming our EPR derived result that the Al-Lewis acid site in the MIL-100(Al) MOF has a similar electron pair acceptor strength as that in the H-ZSM-5 zeolite. Thus our results indicate that the Lewis acidity of Al-sites in MOFs can be reliably probed by the EPR signature of adsorbed NO, verifying its nature by the same time.

\section{CONCLUSION}

In the present work, the application of high resolution pulse EPR methods allowed a detailed characterization of the low temperature NO binding in the MOF MIL-100(Al) confirming only the weak adsorption of $\mathrm{NO}$ in this material, even at the metallic $\mathrm{Al}^{3+}$ sites of the MOF. Results derived from DFT calculations reflect nicely the experimental data and allow a profound understanding of the structural and electronic properties of the EPR detected NO adsorption species NOa binding 
at the regular aluminum framework ion of this MOF. In particular, this methodology might be a notable alternative for the characterization of Lewis acid sites in various Al-based MOFs, using NO as an EPR active probe and simultaneously allowing a certain decision about the nature of those sites. The weak ionic binding of species NOa might explain the failure of a previous IR spectroscopic study to detect its signal at intermediate temperatures $T>173 \mathrm{~K} .{ }^{25}$ Consequently, future IR studies at much lower temperature $T<100 \mathrm{~K}$ are strongly recommended for verification of the presented EPR results. Hopefully future studies will give more insight into the nature of the NO adsorption species NOb observed in the present EPR work. The occurrence of an EPR active NO adsorption species NOc, which most probably interacts with the organic part of the MOF, might be a general situation for MOFs, which all contain organic moieties. Future EPR studies of the NO adsorption on MOFs, having ${ }^{2} \mathrm{H}$ or ${ }^{13} \mathrm{C}$ labelled ligand molecules, should be able to characterize the NO-ligand interaction of this species in more detail.

\section{SUPPLEMENTARY MATERIAL}

See supplementary material for the DFT derived structures of the various cluster models; additional parameters used for the DFT calculations; further results and discussions regarding the $\mathrm{NO}$ adsorption species $\mathrm{NOb}$ and $\mathrm{NOc}$; more figures including an illustration of the $\tau$ and $A$ dependence of blind-spots in a HYSCORE spectrum of a strongly coupled ${ }^{27} \mathrm{Al}$ nucleus; all HYSCORE spectra including the simulations of species Ala, Alb, and Alc; HYSCORE and ENDOR simulations illustrating the effect of the ${ }^{27} \mathrm{Al}$ nqi on the signal of species Ala; an illustration of the orientation selectivity produced by the ${ }^{14} \mathrm{~N}$ hfi for an EPR signal of an NO adsorption species as well as additional tables collecting certain geometric parameters of the various DFT derived cluster models; more DFT derived magnetic parameters calculated with various functionals and different basis-sets; the DFT derived Mulliken and Löwdin reduced spin populations and charges for the cluster models ML3 and M3; and the energy splitting $\Delta$ of species $\mathrm{NOb}$ and NOc.

\section{ACKNOWLEDGMENTS}

The work has been supported by the Deutsche Forschungsgemeinschaft (DFG) within its priority Program No. SPP 1362.

${ }^{1}$ S. Kitagawa and M. Kondo, Bull. Chem. Soc. Jpn. 71, 1739 (1998).

${ }^{2}$ S. Kitagawa, R. Kitaura, and S.-I. Noro, Angew. Chem., Int. Ed. 43, 2334 (2004).

${ }^{3}$ B. Li, M. Chrzanowski, Y. Zhang, and S. Ma, Coord. Chem. Rev. 307, 106 (2016).

${ }^{4}$ M. I. Nandasiri, S. R. Jambovane, B. P. McGrail, H. T. Schaef, and S. K. Nune, Coord. Chem. Rev. 311, 38 (2016).

${ }^{5}$ R. J. Kuppler, D. J. Timmons, Q.-R. Fang, J.-R. Li, T. A. Makal, M. D. Young, D. Yuan, D. Zhao, W. Zhuang, and H.-C. Zhou, Coord. Chem. Rev. 253, 3042 (2009).

${ }^{6}$ U. Mueller, M. Schubert, F. Teich, H. Puetter, K. Schierle-Arndt, and J. Pastré, J. Mater. Chem. 16, 626 (2006).

${ }^{7}$ J. Gascon, A. Corma, F. Kapteijn, and F. X. Llabrés i Xamena, ACS Catal. 4, 361 (2014).

${ }^{8}$ M. Haouas, C. Volkringer, T. Loiseau, G. Férey, and F. Taulelle, J. Phys. Chem. C 115, 17934 (2011).
${ }^{9}$ L. Mitchell, P. Williamson, B. Ehrlichova, A. E. Anderson, V. R. Seymour, S. E. Ashbrook, N. Acerbi, L. M. Daniels, R. I. Walton, M. L. Clarke, and P. A. Wright, Chem. - Eur. J. 20, 17185 (2014).

${ }^{10}$ M. N. Timofeeva, V. N. Panchenko, A. A. Abel, N. A. Khan, I. Ahmed, A. B. Ayupov, K. P. Volcho, and S. H. Jhung, J. Catal. 311, 114 (2014).

${ }^{11}$ T. Rudolf, W. Böhlmann, and A. Pöppl, J. Magn. Reson. 155, 45 (2002).

${ }^{12}$ V. N. Panchenko, M. M. Matrosova, J. Jeon, J. W. Jun, M. N. Timofeeva, and S. H. Jhung, J. Catal. 316, 251 (2014).

${ }^{13}$ M. Gaab, N. Trukhan, S. Maurer, R. Gummaraju, and U. Müller, Microporous Mesoporous Mater. 157, 131 (2012).

${ }^{14}$ M. Chiesa, E. Giamello, and M. Che, Chem. Rev. 110, 1320 (2009).

${ }^{15}$ A. Pöppl, T. Rudolf, P. Manikandan, and D. Goldfarb, J. Am. Chem. Soc. 122, 10194 (2000).

${ }^{16}$ H. Brion, C. Moser, and M. Yamazaki, J. Chem. Phys. 30, 673 (1959).

${ }^{17}$ G. Herzberg and J. W. T. Spinks, Spectra of Diatomic Molecules (D. Van Nostrand Company, New York, 1950).

${ }^{18}$ E. Cartmell and G. W. A. Fowles, Valency and Molecular Structure (Butterworths, London, 1961).

${ }^{19}$ R. P. Orenha and S. E. Galembeck, J. Chem. Educ. 91, 1064 (2014).

${ }^{20}$ C. L. Gardner and M. A. Weinberger, Can. J. Chem. 48, 1317 (1970).

${ }^{21}$ Z. Hu and D. Zhao, CrystEngComm 19, 4066 (2017).

${ }^{22}$ C. Volkringer, H. Leclerc, J.-C. Lavalley, T. Loiseau, G. Férey, M. Daturi, and A. Vimont, J. Phys. Chem. C 116, 5710 (2012).

${ }^{23}$ H. Leclerc, A. Vimont, J.-C. Lavalley, M. Daturi, A. D. Wiersum, P. L. Llwellyn, P. Horcajada, G. Ferey, and C. Serre, Phys. Chem. Chem. Phys. 13, 11748 (2011).

${ }^{24}$ A. Vimont, J.-M. Goupil, J.-C. Lavalley, M. Daturi, S. Surble, C. Serre, F. Millange, G. Ferey, and N. Audebrand, J. Am. Chem. Soc. 128, 3218 (2006).

${ }^{25}$ B. Barth, M. Mendt, A. Pöppl, and M. Hartmann, Microporous Mesoporous Mater. 216, 97 (2015).

${ }^{26}$ C. Volkringer, D. Popov, T. Loiseau, G. Férey, M. Burghammer, C. Riekel, M. Haouas, and F. Taulelle, Chem. Mater. 21, 5695 (2009).

${ }^{27}$ A. Aijaz, T. Akita, H. Yang, and Q. Xu, Chem. Commun. 50, 6498 (2014).

${ }^{28}$ Y. Chen, B. Fan, N. Lu, and R. Li, Catal. Commun. 64, 91 (2015).

${ }^{29}$ R. Grall, T. Hidalgo, J. Delic, A. Garcia-Marquez, S. Chevillard, and P. Horcajada, J. Mater. Chem. B 3, 8279 (2015).

${ }^{30}$ C. Janiak and J. K. Vieth, New J. Chem. 34, 2366 (2010).

${ }^{31}$ L. J. Ignarro and F. Murad, Nitric Oxide. Biochemistry, Molecular Biology, and Therapeutic Implications (Academic Press, San Diego, 1995).

${ }^{32}$ F. J. Corpas and J. B. Barroso, Nitric Oxide 45, 15 (2015).

${ }^{33}$ C. J. Lowenstein, Ann. Intern. Med. 120, 227 (1994).

${ }^{34} \mathrm{G}$. Walford and J. Loscalzo, J. Thromb. Haemostasis 1, 2112 (2003).

${ }^{35}$ F. C. Fang, J. Clin. Invest. 99, 2818 (1997).

${ }^{36}$ A. R. Butler and D. L. H. Williams, Chem. Soc. Rev. 22, 233 (1993).

${ }^{37}$ J.-D. Lio and A. F. Chen, Acta Pharmacol. Sin. 26, 259 (2005).

${ }^{38}$ P. Vallance, Fundam. Clin. Pharmacol. 17, 1 (2003).

${ }^{39}$ S. Huerta, S. Chilka, and B. Bonavida, Int. J. Oncol. 33, 909 (2008).

${ }^{40}$ J. P. Maia-de-Oliveira, C. Trzesniak, I. R. Oliveira, M. J. Kempton, T. M. N. de Rezende, S. Iego, G. B. Baker, S. M. Dursun, J. P. Machado-de-Sousa, and J. E. C. Hallak, Rev. Bras. Psiquiatr. 34(Suppl. 2), 149 (2012).

${ }^{41}$ P. G. Parzuchowski, M. C. Frost, and M. E. Meyerhoff, J. Am. Chem. Soc. 124, 12182 (2002).

${ }^{42}$ F. DeRosa, M. R. Kibbe, S. F. Najjar, M. L. Citro, L. K. Keefer, and J. A. Hrabie, J. Am. Chem. Soc. 129, 3786 (2007).

${ }^{43}$ J. H. Shin, S. K. Metzger, and M. H. Schoenfisch, J. Am. Chem. Soc. 129, 4612 (2007).

${ }^{44}$ J. H. Shin and M. H. Schoenfisch, Chem. Mater. 20, 239 (2008).

${ }^{45}$ D. A. Riccio and M. H. Schoenfisch, Chem. Soc. Rev. 41, 3731 (2012).

${ }^{46}$ P. S. Wheatley, A. R. Butler, M. S. Crane, A. G. Rossi, I. L. Megson, and R. E. Morris, in Molecular Sieves: From Basic Research to Industrial Applications Proceedings of the 3rd International Zeolite Symposium (3rd FEZA), Studies in Surface Science and Catalysis, edited by N. Ž. a. P. N. J. Čejka (Elsevier, 2005), pp. 2033-2040.

${ }^{47}$ P. S. Wheatley, A. R. Butler, M. S. Crane, S. Fox, B. Xiao, A. G. Rossi, I. L. Megson, and R. E. Morris, J. Am. Chem. Soc. 128, 502 (2006).

${ }^{48}$ P. S. Wheatley, A. C. McKinlay, and R. E. Morris, Zeolites and Related Materials: Trends, Targets and Challenges, Proceedings of the 4th International FEZA Conference (Elsevier, 2008), pp. 441-446.

${ }^{49}$ M. Mowbray, X. Tan, P. S. Wheatley, R. E. Morris, and R. B. Weller, J. Invest. Dermatol. 128, 352 (2007).

${ }^{50}$ N. J. Hinks, A. C. McKinlay, B. Xiao, P. S. Wheatley, and R. E. Morris, Microporous Mesoporous Mater. 129, 330 (2010). 
${ }^{51}$ M. J. Ingleson, R. Heck, J. A. Gould, and M. J. Rosseinsky, Inorg. Chem. 48, 9986 (2009).

${ }^{52}$ K. Peikert, L. J. McCormick, D. Cattaneo, M. J. Duncan, F. Hoffmann, A. H. Khan, M. Bertmer, R. E. Morris, and M. Fröba, Microporous Mesoporous Mater. 216, 118 (2015).

${ }^{53}$ E. D. Bloch, W. L. Queen, S. Chavan, P. S. Wheatley, J. M. Zadrozny, R. Morris, C. M. Brown, C. Lamberti, S. Bordiga, and J. R. Long, J. Am. Chem. Soc. 137, 3466 (2015).

${ }^{54}$ F. Bonino, S. Chavan, J. G. Vitillo, E. Groppo, G. Agostini, C. Lamberti, P. D. C. Dietzel, C. Prestipino, and S. Bordiga, Chem. Mater. 20, 4957 (2008).

${ }^{55}$ J. F. Eubank, P. S. Wheatley, G. Lebars, A. C. McKinlay, H. Leclerc, P. Horcajada, M. Daturi, A. Vimont, R. E. Morris, and C. Serre, APL Mater. 2, 124112 (2014).

${ }^{56}$ A. C. McKinlay, J. F. Eubank, S. Wuttke, B. Xiao, P. S. Wheatley, P. Bazin, J.-C. Lavalley, M. Daturi, A. Vimont, G. de Weireld, P. Horcajada, C. Serre, and R. E. Morris, Chem. Mater. 25, 1592 (2013).

${ }^{57}$ C. Kim, S. Diring, S. Furukawa, and S. Kitagawa, Dalton Trans. 44, 15324 (2015).

${ }^{58} \mathrm{~T}$. Li, K. Taylor-Edinbyrd, and R. Kumar, Phys. Chem. Chem. Phys. 17, 23403 (2015).

${ }^{59}$ A. Schweiger and G. Jeschke, Principles of Pulse Electron Paramagnetic Resonance (Oxford University Press, Oxford, UK, New York, 2001).

${ }^{60}$ P. Höfer, A. Grupp, H. Nebenführ, and M. Mehring, Chem. Phys. Lett. 132, 279 (1986).

${ }^{61}$ P. Hofer, J. Magn. Reson., Ser. A 111, 77 (1994).

${ }^{62}$ S. Stoll and A. Schweiger, J. Magn. Reson. 178, 42 (2006).

${ }^{63}$ M. W. Anderson and L. Kevan, J. Chem. Phys. 87, 1 (1987).

${ }^{64}$ F. Neese, Wiley Interdiscip. Rev.: Comput. Mol. Sci. 2, 73 (2012).

${ }^{65}$ C. Lee, W. Yang, and R. G. Parr, Phys. Rev. B 37, 785 (1988).

${ }^{66}$ A. D. Becke, J. Chem. Phys. 98, 5648 (1993).

${ }^{67}$ A. Schäfer, H. Horn, and R. Ahlrichs, J. Chem. Phys. 97, 2571 (1992).

${ }^{68}$ F. Weigend and R. Ahlrichs, Phys. Chem. Chem. Phys. 7, 3297 (2005).

${ }^{69}$ M. Ernzerhof and G. E. Scuseria, J. Chem. Phys. 110, 5029 (1999).

${ }^{70}$ C. Adamo and V. Barone, J. Chem. Phys. 110, 6158 (1999).

${ }^{71}$ C. Adamo, M. Cossi, and V. Barone, J. Mol. Struct.: THEOCHEM 493, 145 (1999).

${ }^{72}$ A. Mavrandonakis, K. D. Vogiatzis, A. D. Boese, K. Fink, T. Heine, and W. Klopper, Inorg. Chem. 54, 8251 (2015).

${ }^{73}$ S. Grimme, S. Ehrlich, and L. Goerigk, J. Comput. Chem. 32, 1456 (2011).

${ }^{74}$ S. Grimme, J. Antony, S. Ehrlich, and H. Krieg, J. Chem. Phys. 132, 154104 (2010).

${ }^{75}$ W. Kutzelnigg, U. Fleischer, and M. Schindler, Deuterium and Shift Calculation, edited by U. Fleischer, W. Kutzelnigg, H.-H. Limbach, G. J. Martin, M. L. Martin, and M. Schindler (Springer Berlin Heidelberg, Berlin, Heidelberg, 1991), pp. 165-262.
${ }^{76} \mathrm{~V}$. Barone, in Recent Advances in Density Functional Methods. Part I, edited by D. P. Chong (World Scientific, 1995).

${ }^{77}$ D. E. Woon and T. H. Dunning, J. Chem. Phys. 98, 1358 (1993).

${ }^{78}$ P. F. Provasi and S. P. A. Sauer, J. Chem. Phys. 133, 54308 (2010).

${ }^{79}$ E. D. Hedegard, J. Kongsted, and S. P. A. Sauer, Phys. Chem. Chem. Phys. 14, 10669 (2012).

${ }^{80}$ J. P. Perdew, K. Burke, and M. Ernzerhof, Phys. Rev. Lett. 77, 3865 (1996).

${ }^{81}$ J. P. Perdew, K. Burke, and M. Ernzerhof, Phys. Rev. Lett. 78, 1396 (1997).

${ }^{82}$ A. D. Becke, Phys. Rev. A 38, 3098 (1988).

${ }^{83}$ J. P. Perdew, Phys. Rev. B 33, 8822 (1986).

${ }^{84}$ A. J. Cohen and N. C. Handy, Mol. Phys. 99, 607 (2001).

${ }^{85}$ N. C. Handy and A. J. Cohen, Mol. Phys. 99, 403 (2001).

${ }^{86}$ J. P. Perdew, J. A. Chevary, S. H. Vosko, K. A. Jackson, M. R. Pederson, D. J. Singh, and C. Fiolhais, Phys. Rev. B 46, 6671 (1992).

${ }^{87}$ J. Tao, J. P. Perdew, V. N. Staroverov, and G. E. Scuseria, Phys. Rev. Lett. 91, 146401 (2003).

${ }^{88}$ J. P. Perdew, Phys. Rev. B 34, 7406 (1986).

${ }^{89}$ J. P. Perdew and W. Yue, Phys. Rev. B 33, 8800 (1986).

${ }^{90}$ S. F. Boys and F. Bernardi, Mol. Phys. 19, 553 (2006).

${ }^{91}$ S. A. Dikanov, A. M. Tyryshkin, and M. K. Bowman, J. Magn. Reson. 144, 228 (2000).

${ }^{92}$ R. I. Samoilova, S. A. Dikanov, A. V. Fionov, A. M. Tyryshkin, E. V. Lunina, and M. K. Bowman, J. Phys. Chem. 100, 17621 (1996).

${ }^{93}$ C. Gemperle and A. Schweiger, Chem. Rev. 91, 1481 (1991).

${ }^{94}$ T. Rudolf, A. Pöppl, W. Brunner, and D. Michel, Magn. Reson. Chem. 37, S93-S99 (1999).

${ }^{95} \mathrm{~F}$. Jensen, Introduction to Computational Chemistry (John Wiley \& Sons, Chichester, England, Hoboken, NJ, 2007).

${ }^{96}$ R. Li, Z. Li, and Y. Pan, Phys. Chem. Miner. 39, 491 (2012).

${ }^{97}$ L. Hermosilla, P. Calle, J. M. Garcia de la Vega, and C. Sieiro, J. Phys. Chem. A 109, 1114 (2005).

${ }^{98}$ A. C. Saladino and S. C. Larsen, J. Phys. Chem. A 107, 1872 (2003).

${ }^{99}$ M. Sundararajan and F. Neese, J. Chem. Theory Comput. 8, 563 (2011).

${ }^{100}$ J. R. Morton and K. F. Preston, J. Magn. Reson. (1969) 30, 577 (1978).

${ }^{101}$ J. H. Enemark and R. D. Feltham, Coord. Chem. Rev. 13, 339 (1974).

${ }^{102}$ C. E. Dinerman and G. E. Ewing, J. Chem. Phys. 53, 626 (1970).

${ }^{103}$ H. Yahiro, A. Lund, and M. Shiotani, Spectrochim. Acta, Part A 60, 1267 (2004).

${ }^{104}$ T. Rudolf, A. Pöppl, W. Hofbauer, and D. Michel, Phys. Chem. Chem. Phys. 3, 2167 (2001).

${ }^{105}$ G. Dousmanis, Phys. Rev. 97, 967 (1955).

${ }^{106}$ P. Wu, Q. Kan, X. Wang, D. Wang, H. Xing, P. Yang, and T. Wu, Appl. Catal., A 282, 39 (2005). 